See discussions, stats, and author profiles for this publication at: https://www.researchgate.net/publication/281117367

\title{
Rigorous asymptotics of traveling-wave solutions to the thin-film equation and Tanner's law
}

Article in Nonlinearity · August 2015

DOI: 10.1088/0951-7715/29/9/2497

CITATIONS

4

3 authors, including:

Lorenzo Giacomelli

Sapienza University of Rome

51 PUBLICATIONS 894 CITATIONS

SEE PROFILE
READS

54

Manuel Victor Gnann

Delft University of Technology

22 PUBLICATIONS 231 CITATIONS

SEE PROFILE 


\section{Max-Planck-Institut für Mathematik in den Naturwissenschaften Leipzig}

Rigorous asymptotics of traveling-wave solutions to the thin-film equation and Tanner's law

\section{by}

\section{Lorenzo Giacomelli, Manuel Gnann, and Felix Otto}

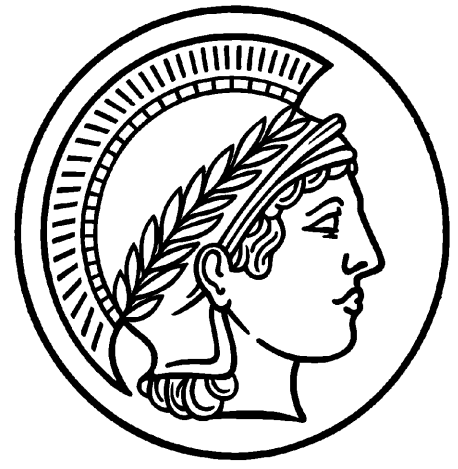





\title{
Rigorous asymptotics of traveling-wave solutions to the thin-film equation and Tanner's law
}

\author{
Lorenzo Giacomelli ${ }^{1}$, Manuel V Gnann ${ }^{2}$, and Felix Otto ${ }^{3}$ \\ ${ }^{1}$ SBAI Department, Sapienza University of Rome, Via A. Scarpa 16, 00161 Roma, \\ Italy \\ 2 Mathematics Department, University of Michigan, 2074 East Hall, 530 Church \\ Street, Ann Arbor, MI 48109-1043 \\ ${ }^{3}$ Max Planck Institute for Mathematics in the Sciences, Inselstr. 22, 04103 Leipzig, \\ Germany \\ E-mail: lorenzo.giacomelli@sbai.uniroma1.it, mvgnann@umich.edu, \\ otto@mis.mpg.de
}

\begin{abstract}
We are interested in traveling-wave solutions to the thin-film equation with zero microscopic contact angle (in the sense of complete wetting without precursor) and inhomogeneous mobility $h^{3}+\lambda^{3-n} h^{n}$, where $h, \lambda$, and $n \in\left(\frac{3}{2}, \frac{7}{3}\right)$ denote film height, slip length, and mobility exponent, respectively. Existence and uniqueness have been established by Maria Chiricotto and the first of the authors in previous work under the assumption of sub-quadratic growth as $h \rightarrow \infty$.

In the present note we investigate the asymptotics of solutions as $h \searrow 0$ (the contact-line region) and $h \rightarrow \infty$. As $h \rightarrow \infty$ we identify, to leading order, the logarithmic Tanner profile - i.e., the solution to the corresponding unperturbed problem with $\lambda=0$ - that determines the apparent macroscopic contact angle. Besides higher-order terms, corrections turn out to affect the asymptotic law as $h \rightarrow \infty$ only by setting the length scale in the logarithmic Tanner profile. Moreover, we prove that both the correction and the length scale depend smoothly on $n$. Hence, in line with the common philosophy, the precise modeling of liquid-solid interactions (within our model, the mobility exponent) does not affect the qualitative macroscopic properties of the film.
\end{abstract}

AMS classification scheme numbers: 35K65, 34B16, 34B40, 34C45, 34E10, 76A20

Keywords: Degenerate parabolic equations, Singular nonlinear boundary value problems, Boundary value problems on infinite intervals, Invariant manifolds, Perturbations, asymptotics, Thin fluid films

Submitted to: Nonlinearity 


\section{Introduction}

We consider the following free boundary problem to the thin-film equation:

$$
\begin{array}{ll}
\partial_{t} h+\partial_{z}\left(\left(h^{3}+\lambda^{3-n} h^{n}\right) \partial_{z}^{3} h\right)=0 & \text { for } t>0 \text { and } z>Z(t), \\
h=\partial_{z} h=0 & \text { for } t>0 \text { and } z=Z(t), \\
\lim _{z \rightarrow Z(t)^{+}}\left(h^{2}+\lambda^{3-n} h^{n-1}\right) \partial_{z}^{3} h=\frac{\mathrm{d} Z}{\mathrm{~d} t}(t) & \text { for } t>0,
\end{array}
$$

The function $h=h(t, z)$ describes the height of a liquid viscous thin film on a flat solid as a function of time $t \geq 0$ and position $z \in \mathbb{R}$. For simplicity we assume translation invariance in one dimension (perpendicular to the plane spanned by the $z$-and $h$-axis), which is why the base point $z$ is just a real number. We assume that the film infinitely extends on the interval $z \in(Z(t), \infty)$ and has a free boundary at $z=Z(t)$, to which we refer to as the "contact line" or "triple junction" (since liquid, gas, and solid meet here). Then the first condition in $(1 b)$ just determines the position of $Z(t)$. The second leads to a zero (equilibrium or microscopic) contact angle at the intersection of the interfaces liquid-gas and liquid-solid. Condition $(1 c)$ is a kinematic one: Rewriting (1a) as a continuity equation for the film height $\partial_{t} h+\partial_{z}(V h)=0$ in $\{h>0\}$, the transport velocity of $h$ (this is the vertically averaged velocity of the fluid) is given by $V=\left(h^{2}+\lambda^{3-n} h^{n-1}\right) \partial_{z}^{3} h$ and, on approaching the contact line, has to match the velocity $\frac{\mathrm{d} Z}{\mathrm{~d} t}$ of the free boundary.

Problem (1) contains two parameters, namely the slip length $\lambda$ and the mobility exponent $n$, both depending on the modeling of liquid-solid interactions. For $\lambda=0$ solutions to (1) exhibit the known no-slip paradox: The solution is singular at the free boundary $z=Z(t)$ and viscous dissipation is unbounded $[16,10]$. Physically $\lambda=0$ corresponds to the assumption of no slip at the substrate. A way to restore a continuum description is to introduce the length scale $\lambda>0$, corresponding to (in general nonlinear) slip conditions $[15,40]$. In mathematical terms, equation $(1 a)$ degenerates at $z=Z(t)$ (i.e. it is not uniformly parabolic) and the addend $\sim h^{n}$ in the mobility $h^{3}+\lambda^{3-n} h^{n}$ relaxes this degeneracy if $n<3$. We will assume $n \in\left(\frac{3}{2}, \frac{7}{3}\right)$, which contains the physically relevant case $n=2$ : In fact, the model for $n=2$ can be derived by means of asymptotic expansions (known as lubrication approximation) from the underlying Navier-Stokes equations with a (linear) Navier-slip condition at the substrate (see e.g. $[6,32])$. We refer to [26] for a rigorous justification of the Navier-slip condition, first

proposed by Navier in his seminal work in [31], and to [21, 29, 30] for rigorous derivations of the lubrication approximation in a related framework.

\section{Traveling waves}

As outlined by Hocking in [25] (cf. [6, 9] for more recent accounts), there is an intermediate region between the contact-line region, where the shape of the film is governed by a balance of surface tensions (Young's law) determining the equilibrium (microscopic) contact angle, and the interior of the film, where the equilibrium shape 
forms a parabola (constant mean curvature in lubrication approximation). It is in this intermediate region that the apparent (macroscopic) contact angle is determined. The aim of this note is to precisely investigate this region and its dependence on the physical assumptions for the liquid-solid interactions.

Therefore, it is convenient to assume a simplified situation, in which we neglect the interior of the droplet by considering a traveling wave $h(t, z)=H(x)$ with $x=z+V t$ (where $H(x)$ is a fixed profile) propagating with constant speed $-V<0$ to the left. We can insert this ansatz into equation $(1 a)$ and integrate once, thereby employing the boundary conditions $(1 b)$ and $(1 c)$, so that the resulting equation reads

$$
\left(H^{2}+\lambda^{3-n} H^{n-1}\right) \frac{\mathrm{d}^{3} H}{\mathrm{~d} x^{3}}=-V .
$$

By rescaling $x$ and $H$, we can assume without loss of generality $V=\frac{1}{3}$ (a convenient choice in view of the asymptotics as $x \rightarrow \infty)$ and $\lambda=1$. This leads to

$$
\begin{array}{ll}
\left(H^{2}+H^{n-1}\right) \frac{\mathrm{d}^{3} H}{\mathrm{~d} x^{3}}=-\frac{1}{3} & \text { for } x>0, \\
H=\frac{\mathrm{d} H}{\mathrm{~d} x}=0 & \text { at } x=0,
\end{array}
$$

where, by translation in $x$, without loss of generality the contact line is assumed to obey $Z(0)=0$, i.e. $Z(t)=-\frac{1}{3} t$.

\section{Dominant behavior as $x \rightarrow \infty$}

Obviously, problem (2) is lacking a third boundary condition in order to allow for existence of a unique solution. In [8], Chiricotto and one of the authors provef that problem (2) admits a unique solution in the class $C^{1}([0, \infty)) \cap C^{3}((0, \infty))$ (i.e. a classical solution) such that

$$
\frac{\mathrm{d}^{2} H}{\mathrm{~d} x^{2}} \rightarrow 0 \quad \text { as } x \rightarrow \infty
$$

In that case, simple asymptotic considerations suggest that $H$ approximately obeys the third-order equation

and that

$$
H^{2} \frac{\mathrm{d}^{3} H}{\mathrm{~d} x^{3}}=-\frac{1}{3}
$$

$$
H=x(\ln x)^{\frac{1}{3}}(1+o(1)) \quad \text { as } x \rightarrow \infty .
$$

Note that (4) was solved implicitly in terms of Airy functions by Duffy and Wilson in [14], thus making the asymptotic result (5) rigorous on the level of the unperturbed problem (4). Formally differentiating (5) (or using the explicit result in [14]) and undoing the normalization of the speed $V$, one may derive

$$
\left(\frac{\mathrm{d} H}{\mathrm{~d} x}\right)^{3}=3 V \ln x(1+o(1)) \quad \text { as } x \rightarrow \infty .
$$

$\ddagger$ Note that there only the case $n=2$ is considered. However, it is apparent that the precise value of $n \in\left(\frac{3}{2}, \frac{7}{3}\right)$ is immaterial for the analysis. 
Relation (6) may be interpreted by saying that, in complete wetting, the speed of the contact line (which in our case is identical to the speed of the traveling wave) is proportional, up to a logarithmic correction, to the cube of the apparent (macroscopic) contact angle, a fact which is often referred to as Tanner's law [39] (a more general relation including partial wetting is also referred to as the Cox-Hocking relation [12, 24]). For this reason, we shall hereafter refer to (5) or (6) as "Tanner's law".

We remark that (4) is invariant with respect to translations in $x$ and the scaling $(H, x) \mapsto(B H, B x)$ for any $B>0$. Up to these two transformations, the solution of (4) meeting (3) is uniquely determined, that is, selecting an arbitrary solution $H$ of $(3) \&(4)$, any solution to $(3) \&(4)$ can be written as

$$
B^{-1} H(B(x+c)) \text {, where } B, c>0 \text { are free parameters. }
$$

\section{Dominant behavior as $x \searrow 0$}

As $x \searrow 0$ (i.e. $H \searrow 0$ ), the term $H^{n-1}$ dominates in the bracket of $(2 a)$ and one expects the leading order-behavior of (2) to be determined by the unperturbed problem

$$
\begin{array}{ll}
H^{n-1} \frac{\mathrm{d}^{3} H}{\mathrm{~d} x^{3}}=-\frac{1}{3} & \text { for } x>0 \\
H=\frac{\mathrm{d} H}{\mathrm{~d} x}=0 & \text { at } x=0 .
\end{array}
$$

Problem (7) was studied in detail in [5]. Since we only have two boundary conditions (7b) for a third-order ODE (7a), we need an additional condition to uniquely determine $H$. As problem (7) is invariant under the rescaling

$$
(H, x) \mapsto\left(c H, c^{\frac{n}{3}} x\right) \text { for any } c>0,
$$

we look for solutions $H$ to (7) that are invariant with respect to this scaling transformation, that is,

$$
H(x):=H_{\mathrm{TW}}(x)=c^{-\frac{3}{n}} H_{\mathrm{TW}}(c x) \text { for any } c>0 \text { and all } x>0 .
$$

Setting $c:=x^{-1}$, this amounts to having $H_{\mathrm{TW}}=H_{\mathrm{TW}}(1) x^{\frac{3}{n}}$, where $H_{\mathrm{TW}}(1)$ can be determined by using $(7 a)$. Thus we arrive at a solution of the form

$$
H_{\mathrm{TW}}(x)=(3 A)^{-\frac{1}{n}} x^{\frac{3}{n}} \quad \text { for } x>0, \quad \text { where } A:=\frac{3}{n}\left(\frac{3}{n}-1\right)\left(2-\frac{3}{n}\right) .
$$

The dependence of the constant $A$ on $n$ clearly indicates the (known) interval $n \in\left(\frac{3}{2}, 3\right)$. The further restriction $n<\frac{7}{3}$ is due to a higher resonance for $n=\frac{7}{3}$, so that a weak singularity of the model occurs, which to our knowledge was not known before (cf. (25)\&(26) and the discussion thereafter). While the leading-order behavior of $H$ as $x \searrow 0$ is transparent, the corrections of this result are more involved. We will use ideas developed in [19] to address this issue. 


\section{Coordinate transformations}

Obviously problem $(2 a)$ is translation invariant in $x$. This symmetry enables us to perform another trivial integration. We first notice that the solution $H$ of $(2) \&(3)$ obeys

$$
H>0 \text { for all } x>0 .
$$

Using equation $(2 a)$ we have

$$
\frac{\mathrm{d}^{3} H}{\mathrm{~d} x^{3}}<0 \text { for all } x>0 \text {. }
$$

Due to the boundary condition (3), this implies

$$
\frac{\mathrm{d}^{2} H}{\mathrm{~d} x^{2}}>0 \text { for all } x>0 .
$$

Using the second boundary condition in $(2 b)$, that is, $\frac{\mathrm{d} H}{\mathrm{~d} x}=0$ at $x=0$, this amounts to

$$
\frac{\mathrm{d} H}{\mathrm{~d} x}>0 \text { for all } x>0 .
$$

Hence $H$ is a strictly monotone function and so we can as well formulate our equation in terms of the position $x$ as a function of the height $H$, thus getting rid of the translation invariance. In fact, it is more convenient to consider

$$
\psi:=\left(\frac{\mathrm{d} H}{\mathrm{~d} x}\right)^{2}>0 \text { as a function of } H
$$

as our new unknown§. Observe

$$
\begin{aligned}
& \frac{\mathrm{d} \psi}{\mathrm{d} H}=2 \frac{\mathrm{d}^{2} H}{\mathrm{~d} x^{2}} \stackrel{(11)}{>} 0, \\
& \frac{\mathrm{d}^{2} \psi}{\mathrm{d} H^{2}}=2\left(\frac{\mathrm{d} H}{\mathrm{~d} x}\right)^{-1} \frac{\mathrm{d}^{3} H}{\mathrm{~d} x^{3}} \stackrel{(10),(12)}{<} 0 .
\end{aligned}
$$

Equation $(2 a)$ turns into

$$
\frac{\mathrm{d}^{2} \psi}{\mathrm{d} H^{2}}+\frac{1}{\sqrt{\psi}} \phi(H)=0 \quad \text { for } H>0, \quad \text { where } \phi(H):=\frac{2}{3\left(H^{2}+H^{n-1}\right)} .
$$

Because of (13), the second boundary condition in $(2 b)$ translates into

$$
\psi=0 \quad \text { at } H=0
$$

and due to (14a), condition (3) now reads

$$
\frac{\mathrm{d} \psi}{\mathrm{d} H} \rightarrow 0 \quad \text { as } H \rightarrow \infty
$$

Notably, the boundary conditions remain linear after transforming as in (13). Additionally, only the second derivative $\frac{\mathrm{d}^{2} \psi}{\mathrm{d} H^{2}}$ and the function $\psi$ itself appear in (15). The main result of [8] is that problem (15) admits a unique classical solution (see footnote $\ddagger)$, i.e. a unique solution $\psi \in C^{0}([0, \infty)) \cap C^{2}((0, \infty))$. From now on, we will focus on discussing problem (15).

$\S$ Our transformations are similar to those used in $[3,5,7,41]$, mainly capitalizing on the translation invariance of the problem by using $H$ or $\ln H$ as an independent variable. 
Dominant behavior as $H \rightarrow \infty$

In view of (13), the asymptotic expression (5) implies

$$
\psi=\left(\frac{\mathrm{d} H}{\mathrm{~d} x}\right)^{2}=(\ln x)^{\frac{2}{3}}(1+o(1)) \quad \text { as } x \rightarrow \infty
$$

for a solution of

$$
\frac{\mathrm{d}^{2} \psi}{\mathrm{d} H^{2}}+\frac{2}{3} \psi^{-\frac{1}{2}} H^{-2}=0 \text { for } H>0
$$

subject to

$$
\frac{\mathrm{d} \psi}{\mathrm{d} H} \rightarrow 0 \quad \text { as } H \rightarrow \infty
$$

Using $x(H) \stackrel{(5)}{=} H(\ln H)^{-\frac{1}{3}}(1+o(1))$ as $H \rightarrow \infty$, one obtains

$$
\psi:=\left(\frac{\mathrm{d} H}{\mathrm{~d} x}\right)^{2}=(\ln H)^{\frac{2}{3}}(1+o(1)) \quad \text { as } H \rightarrow \infty .
$$

Now we obtain a one-parameter family of solutions $\psi(B H)$ with a free parameter $B>0$, where $\psi>0$ is an arbitrary solution to (16). A more detailed analysis of corrections to (17), contained in Section 4 and Section 5, shows the asymptotic expansion

$$
\psi=(\ln H)^{\frac{2}{3}}-\frac{2}{9}(\ln \ln H)(\ln H)^{-\frac{1}{3}}-\frac{2 \ln B}{3}(\ln H)^{-\frac{1}{3}}+o\left((\ln H)^{-\frac{1}{3}}\right)
$$

as $H \rightarrow \infty$, so that a unique solution $\psi=\psi_{\mathrm{T}} \in C^{2}$ is selected (cf. Proposition 3.1) by enforcing

$$
\psi_{\mathrm{T}}=(\ln H)^{\frac{2}{3}}-\frac{2}{9}(\ln \ln H)(\ln H)^{-\frac{1}{3}}+o\left((\ln H)^{-\frac{1}{3}}\right) \quad \text { as } H \rightarrow \infty
$$

\section{Dominant behavior as $H \searrow 0$}

Since $\phi(H)=\frac{2}{3} H^{1-n}(1+o(1))$ as $H \searrow 0$, one expects the leading-order behavior of $(15 a) \&(15 b)$ to be determined by (cf. (7))

$$
\begin{array}{ll}
\frac{\mathrm{d}^{2} \psi}{\mathrm{d} H^{2}}+\frac{2}{3} H^{1-n} \psi^{-\frac{1}{2}}=0 & \text { for } H>0, \\
\psi(H)=0 & \text { at } H=0 .
\end{array}
$$

Analogous to the treatment of problem (7), we need an additional condition to select a single solution. The scaling invariance of (19) suggests to assume

$$
\psi(H):=\psi_{\mathrm{TW}}(H)=c^{-2+\frac{2}{3} n} \psi_{\mathrm{TW}}(c H) \text { for any } c>0 .
$$

Setting $c:=H^{-1}$, we have $\psi_{\mathrm{TW}}(H)=\psi_{\mathrm{TW}}(1) H^{2-\frac{2}{3} n}$ and using (19a), we get (cf. (9)):

$$
\psi_{\mathrm{TW}}(H)=C H^{2-\frac{2}{3} n} \quad \text { for } H>0 \text {, with } C:=3^{\frac{2}{3}}((3-n)(2 n-3))^{-\frac{2}{3}} .
$$

We will discuss corrections to this leading-order behavior in Section 6 and Section 7. 


\section{Notation}

We write $f \gtrsim g$ (of $g \lesssim f$ ) whenever a constant $C>0$, only depending on $n$, exists such that $f \geq C g$. We say that a property is true for $f \gg 1$ (or $f \ll 1$ ) whenever a constant $C>0$, only depending on $n$, exists such that the property is true for $f \geq C$ (or $f \leq \frac{1}{C}$ ).

For a Banach space $F$ and a map $\mathcal{G}: F \supset U \rightarrow F$ we write $\partial_{F} \mathcal{G}[f] \partial f$ for the Gâteaux derivative of $\mathcal{G}$ in $(f, \partial f) \in U \times F$.

We write $f(x)=O(g(x))$ as $x \rightarrow x_{0}$ whenever $\limsup _{x \rightarrow x_{0}}\left|\frac{f(x)}{g(x)}\right|<\infty$ and $f(x)=o(g(x))$ whenever $\lim _{x \rightarrow x_{0}}\left|\frac{f(x)}{g(x)}\right|=0$.

\section{The main result}

The aim of this note is to rigorously derive the asymptotic behavior of $\psi$ as $H \searrow 0$ and $H \rightarrow \infty$. Furthermore, we are interested in investigating the dependence of the asymptotic expressions on the mobility exponent $n$. This can be formulated in the following statement:

Theorem 2.1. Let $n \in\left(\frac{3}{2}, \frac{7}{3}\right)$. The unique classical solution $\psi=\psi(H)$ of problem (15) obeys the following asymptotic behavior: There exists a parameter $B$ and a function $R(H)$ such that

$$
\psi(H)=\psi_{\mathrm{T}}(B H)(1+R(H)) \quad \text { for } B H \gg 1,
$$

where

$$
R(H)=O\left((\ln H)^{-1} H^{-(3-n)}\right) \quad \text { as } H \rightarrow \infty .
$$

Both $B$ and locally in $H$ also $R$ are $C^{1}$-functions of $n$. Furthermore,

$$
\psi(H)=C H^{2-\frac{2}{3} n}(1+o(1)) \quad \text { as } H \searrow 0,
$$

where $C:=3^{\frac{2}{3}}((3-n)(2 n-3))^{-\frac{2}{3}}$ (cf. (21)).

Remark 2.2. In terms of the unique solution $H$ to (2)E(3), Theorem 2.1 implies in particular that

$$
\left(\frac{\mathrm{d} H}{\mathrm{~d} x}\right)^{3} \stackrel{(13)}{=}(\psi(H))^{\frac{3}{2}} \stackrel{(18),(22)}{=} \ln (B H)-\frac{1}{3} \ln \ln (B H)+o(1) \quad \text { as } x \rightarrow+\infty(24)
$$

with $B$ a $C^{1}$-function of $n$. Note that (24) improves (6) by setting the length scale in the logarithmic Tanner profile.

Discussion and comparison to other works

The essentially new insight of Theorem 2.1 is that, up to rescaling with the $C^{1}$-function $B=B(n)$, the exponent $n$ (determining the boundary condition at the substrate) has no significant effect on the leading-order asymptotic behavior of $\psi$ as $H \rightarrow \infty$. The first $n$ dependent correction in the bracket in $(22)$ is of order $\sim(\ln H)^{-1} H^{-(3-n)}$. Furthermore, 
we prove an infinitesimal statement, that is, $C^{1}$-variations of the mobility (by varying the exponent $n$ ) lead to a $C^{1}$-change of $B$ and the solution.

It appears that our method (detailed below) is applicable also to other mobility exponents, contact lines with non-zero contact angle, or thin films with precursor. Our prediction is that the structural result (22) is true in these cases as well: We expect that Tanner's law (5)\&(6) is in general only perturbed through the physics of liquidsolid interactions by a length scale and a higher-order correction - both of which have a $C^{1}$-dependence on variations of the mobility exponent, the slip length or the contact angle (at least within the partial wetting regime), or the disjoining pressure. This is in agreement with the physical intuition that the precise modeling of liquid-solid interactions has no significant effect on the macroscopic properties of the thin film, as suggested by the considerations of Hocking [25], respectively DeGennes [13] and Eggers and Stone [17] (see also [2]): for instance, the parameter $B$ in Theorem 2.1, part (b) is used as a matching parameter in the formal asymptotic expansions of $[17,25]$ (Hocking in [25, Sec. 4] even calculates a numerical approximation for $n=2$ ).

In this respect, it is worth mentioning that, instead, the physics at the contact line do have a significant effect on the macroscopic properties of the thin film (as may be easily understood by comparing steady states with different equilibrium contact angles). Here, efforts are being recently undertaken towards continuum modeling of frictional forces at the contact line [35, 36, 37] (see also [34]), leading to contact-line conditions relating speed and microscopic contact angle. Formal asymptotic results in [9] suggest that, also for these models, the precise modeling of liquid-solid interactions corrects the macroscopic properties of the flow only logarithmically.

Besides the already mentioned ones [9, 25], our result is closely related to quite a few other works in which Tanner's law is addressed, such as $[1,4,14,18,22,28,27]$. At the level of the PDE (1a), the only previous rigorous work is by two of the authors [20], where the effect of slippage on the spreading rate of the apparent support $\{h>\lambda\}$ of solutions to (1) is investigated: Using integral estimates on physical quantities, Tanner's law is demonstrated in an intermediate (in time) region (where the dynamics are neither governed by the initial data nor by the physics due to slippage). However, none of the aforementioned contributions rigorously addresses either (22) (i.e. (24)) or the dependence of logarithmic corrections on the mobility exponent (in fact, to our knowledge none of the previous contributions even rigorously derive Tanner's law (5)\&(6) for the perturbed problem (15), see Section 4). The Duffy-Wilson setting in [14] - characterizing the solution of the unperturbed problem (16) by an equation involving explicit functions - does not seem to allow for a perturbation argument as stated in Theorem 2.1, part (b).

We mention that it was already suggested in [19] to study traveling-wave solutions to the two-dimensional Stokes problem for a moving cusp. We expect two asymptotic regimes (the contact-line region and the Tanner region) for this problem as well. Our hope is to 
rigorously recover the lubrication limit on the level of the traveling wave as it was done for Darcy's flow in the Hele-Shaw cell in [20, 29, 30].

Two-variable analyticity and the limitation $n<7 / 3$

In fact, we are able to prove a stronger result than (23), that is,

$$
\psi(H)=C H^{2-\frac{2}{3} n}\left(1+\bar{\mu}\left(H^{3-n}, H^{\alpha}\right)\right) \quad \text { for } 0 \leq H \ll 1,
$$

where

$$
\alpha:=\frac{1}{6} \sqrt{-27+36 n-8 n^{2}}-\frac{3}{2}+\frac{2}{3} n
$$

and $\bar{\mu}=\bar{\mu}\left(y_{1}, y_{2}\right)$ is analytic in a neighborhood of $\left(y_{1}, y_{2}\right)=(0,0)$. The exponents $\alpha$ and $3-n$ are related to the linearization of $(15 a)$ around $\psi / \psi_{\mathrm{T} W}=1$, see Sections 6 and 7. This two-variable analyticity was already conjectured by the authors in [19], where source-type self-similar solutions of

$$
\partial_{t} h+\partial_{z}\left(h^{n} \partial_{z}^{3} h\right)=0 \text { for } z \in\{h>0\}
$$

with $n \in\left(\frac{3}{2}, 3\right)$ and subject to $h=\partial_{z} h=0$ at $z \in \partial\{h>0\}$ (cf. (1b)) have been investigated. There indeed $h(t, z)=t^{-\frac{1}{n+4}} H_{\mathrm{s}}(x)$ with $x:=t^{-\frac{1}{n+4}} z$ and $H_{\mathrm{s}}(x)=$ $H_{\mathrm{TW}}(x)\left(1+v_{\mathrm{s}}\left(x, x^{\beta}\right)\right)$, where $H_{\mathrm{TW}}$ is defined as in $(21), v_{\mathrm{s}}\left(x_{1}, x_{2}\right)$ is an analytic function in a neighborhood of $(x, y)=(0,0)$, and $\beta=\frac{3}{n} \alpha, \alpha$ being the same as in(26).

Expansion (26) can be proven with similar methods as in [19]. There a direct method treating the corresponding nonlinear third-order ODE was detailed and a dynamical system's approach was only briefly sketched as a possible alternative strategy (cf. [19, Sec. 2.2]). For the latter approach, the authors reduced the problem to the study of an invariant manifold of the dynamical system $\left(x, F, F^{\prime}, F^{\prime \prime}\right)$, where $F:=\frac{H_{\mathrm{s}}}{H_{\mathrm{TW}}}$, $F^{\prime}:=x \frac{\mathrm{d}}{\mathrm{d} x} F$, and $F^{\prime \prime}:=\left(x \frac{\mathrm{d}}{\mathrm{d} x}\right)^{2} F$. Setting $s:=\ln x$, the dynamics close to the contact line $s=-\infty$ are then determined by all trajectories emanating from the hyperbolic stationary point $\left(x, F, F^{\prime}, F^{\prime \prime}\right)=(0,1,0,0)$, that is, they have to lie on the unstable manifold tangent to the two-dimensional unstable eigenspace, which is spanned by the eigenvectors corresponding to the eigenvalues 1 and $\beta$, respectively. Thus one can lift the system to the unstable manifold, locally parametrized by coordinates $\left(F_{1}, F_{2}\right)$, that is, one needs to solve the ODE

$$
\left(\frac{\mathrm{d}}{\mathrm{d} s}-\left(\begin{array}{ll}
1 & 0 \\
0 & \beta
\end{array}\right)\right) \cdot\left(\begin{array}{c}
F_{1} \\
F_{2}
\end{array}\right)=g\left(F_{1}, F_{2}\right),
$$

where $g\left(F_{1}, F_{2}\right)=O\left(F_{1}^{2}+F_{2}^{2}\right)$ is an analytic nonlinear correction. It was then argued that by the contraction-mapping theorem for given data $\left(F_{1}(0), F_{2}(0)\right)$ a unique solution exists. However, the authors did not provide a proof for the claim that $F_{j}=\bar{F}_{j}\left(e^{s}, e^{\beta s}\right)$, where $\bar{F}_{j}\left(x_{1}, x_{2}\right)$ are analytic functions in $\left\{\left|x_{1}\right|+\left|x_{2}\right| \ll 1\right\}$. In fact, this appears to be nontrivial as for $n \in\left(\frac{3}{2}, 3\right)$, the exponent $\beta$ covers the interval $(0,1)$ and for $\beta=\frac{1}{k}$ with an integer $k \geq 2$ a resonant term $\sim F_{2}^{k}$ in the nonlinearity $g\left(F_{1}, F_{2}\right)$ should be expected. Inverting the linear part of (27) from $s=-\infty$ (or $\left(x_{1}, x_{2}\right)=(0,0)$, respectively), these 
resonant terms in the nonlinearity are not integrable and a fixed-point argument is doomed to fail.

A way how to close this gap and to restore the validity of the arguments is to parametrize the unstable manifold as a graph $F^{\prime}=F^{\prime}(x, u)$ with $u:=F-1$, that is,

$$
x \frac{\mathrm{d} u}{\mathrm{~d} x}=G(x, u),
$$

where $G=G(x, u)$ is analytic in $\{|x|+|u| \ll 1\}$ with $G(0,0)=0$. Knowing $\frac{\partial G}{\partial u}(0,0)=\beta$ from the parametrization of the tangent space, we can reformulate (28) as

$$
\left(x \frac{\mathrm{d}}{\mathrm{d} x}-\beta\right) u=G(x, u)-\beta u,
$$

where the right-hand side is now quadratic in $u$. Unfolding this problem, by replacing $u$ with $\bar{u}\left(x_{1}, x_{2}\right)$ and $x \frac{\mathrm{d}}{\mathrm{d} x}$ with $x_{1} \partial_{x_{1}}+\beta x_{2} \partial_{x_{2}}$, we may then solve

$$
\left(x_{1} \partial_{x_{1}}+\beta x_{2} \partial_{x_{2}}-\beta\right) \bar{u}=G\left(x_{1}, \bar{u}\right)-\beta \bar{u},
$$

leading to the fixed-point problem

$$
\bar{u}\left(x_{1}, x_{2}\right)=-b x_{2}+\int_{0}^{1} r^{-\beta}\left(G\left(r x_{1}, \bar{u}\left(r x_{1}, r^{\beta} x_{2}\right)\right)-\beta \bar{u}\left(r x_{1}, r^{\beta} x_{2}\right)\right) \frac{\mathrm{d} r}{r},(29)
$$

with a parameter $b \in \mathbb{R}$ (in fact $b>0$ can be proven, cf. [19, Sec. 5]). As detailed for a similar case in $[19$, Sec. 3, 4], the contraction-mapping theorem yields for every $b \in \mathbb{R}$ and sufficiently small $\varepsilon>0$ the existence of an analytic solution $\bar{u}$ to (29).

In the present note, we will employ the same approach, but for simplicity we restrict ourselves to the leading-order asymptotics only. Notably for $n=\frac{7}{3}$, a resonance of the exponents (26) in (25) occurs $\left(\alpha=3-n=\frac{2}{3}\right)$. In this case, (25) ceases to be true and we expect logarithmic corrections in $H$ to be present. This is reflected by the fact that a diagonalization of the respective dynamical system is not possible anymore and a two-dimensional Jordan block in the normal form of the linearized evolution is present. As we are mainly interested in a perturbative result of $n=2$ (Navier slip), our analysis is restricted to the interval $n \in\left(\frac{3}{2}, \frac{7}{3}\right)$.

\section{Proof of the main result: a transversality argument}

\section{Outline}

Our strategy to prove Theorem 2.1 is as follows: We first construct two one-parametric solution manifolds (hence two-dimensional manifolds in phase space) for $H \gg 1$ (Section 5 ) and $H \ll 1$ (Section 7 ) of the three-dimensional dynamical system $\left(H, \psi, \frac{\mathrm{d} \psi}{\mathrm{d} H}\right)$ associated to $(15 a)$. In Section 4 and Section 6 we additionally demonstrate that the unique solution of (15) lies on these manifolds. In other words: The manifolds intersect in a unique curve that defines the solution of (15). Then we prove that these manifolds intersect transversally. This yields the $C^{1}$-dependence of $B$ on $n$. 
Characterization of the solution manifolds

We are able to characterize a one-parametric solution manifold of $(15 a) \&(15 c)$ (the "intermediate" region, where Tanner's law dominates):

Proposition 3.1. For every $B>0$ there exists a function $R_{B}(H)$ for $H \gg 1+B^{-1}$ such that

$$
\psi_{B}(H)=\psi_{\mathrm{T}}(B H)\left(1+R_{B}(H)\right) \quad \text { for } H \gg 1+B^{-1},
$$

defines a solution to (15a) $\mathcal{G}(15 \mathrm{c})$, where $\psi_{\mathrm{T}}=\psi_{\mathrm{T}}(H)$ is the unique classical solution to (16)E(18), and

$$
R_{B}(H) \lesssim B^{3-n}(\ln H)^{-1} H^{-(3-n)} \quad \text { for } H \gg 1+B^{-1} .
$$

The correction $R_{B}(H)$, locally in $H$, has a $C^{1}$-dependence on $B$ and $n$ and the boundary condition

$$
\partial_{H} \partial_{B} \psi_{B}=-\frac{2}{9 B}(\ln H)^{-\frac{4}{3}} H^{-1}(1+o(1)) \quad \text { as } H \rightarrow \infty
$$

is satisfied. Furthermore, there exists a $B>0$ such that the unique solution $\psi$ of problem (15) coincides with $\psi_{B}$.

We prove this proposition in Section 4 and Section 5. The approach mainly relies on the application of the contraction-mapping and the implicit function theorem of a suitably transformed system, in which the dependence on the parameter $B$ is more transparent. For film heights $H \ll 1$ we are able to show an analogous result:

Proposition 3.2. For every $b \in \mathbb{R}$ there exists a function $\mu_{b}=\mu_{b}(H)$ for $H \ll$ $(1+|b|)^{-\frac{1}{\alpha}}$ such that

$$
\psi_{b}(H)=C H^{2-\frac{2}{3} n}\left(1+\mu_{b}(H)\right) \quad \text { for } H \ll(1+|b|)^{-\frac{1}{\alpha}}
$$

defines a solution to (15a)G(15b), where $C$ is defined in (21) and

$$
\mu_{b}(H)=b H^{\alpha}\left(1+O\left(H^{\delta}\right)\right) \quad \text { as } H \searrow 0,
$$

with $\alpha$ given by (26) and $\delta:=\min \{3-n-\alpha, \alpha\}$. The function $\mu_{b}$ (and thus also $\psi_{b}$ ) depends smoothly on $n \in\left(\frac{3}{2}, \frac{7}{3}\right)$ and $b \in \mathbb{R}$ and the boundary condition

$$
\partial_{b} \psi_{b}=C H^{2-\frac{2}{3} n+\alpha}\left(1+O\left(H^{\alpha}\right)\right) \quad \text { as } H \searrow 0
$$

holds true. Furthermore, the unique solution to (15) has the structure (31) for some $b \in \mathbb{R}$.

The proof relies on the study of an invariant manifold of a suitable dynamical system and is detailed in Section 6 and Section 7. 


\section{Transversality}

In Propositions 3.1 and 3.2 we have constructed two one-parameter families of solutions $\left(\psi_{b}\right)_{b \in \mathbb{R}}$ and $\left(\psi_{B}\right)_{B>0}$ fulfilling the boundary conditions (cf. $(15 b)$ )

$$
\psi_{b}=0 \text { at } H=0
$$

and (cf. $(15 c))$

$$
\partial_{H} \psi_{B} \rightarrow 0 \text { as } H \rightarrow \infty,
$$

respectively. Differentiating $\psi_{b}$ with respect to $b$, condition $(32 a)$ remains satisfied (cf. $(31 c)$ ). Additionally, $\partial_{B} \psi_{B}$ meets condition (32b) (cf. (30c)). If $b, B \in \mathbb{R}$ are chosen such that $\psi_{b}=\psi_{B}=\psi$, where $\psi$ is the unique classical solution to (15), then $\partial_{b} \psi_{b}$ and $\partial_{B} \psi_{B}$ exist globally as well and satisfy the linear equation

$$
\frac{\mathrm{d}^{2} \eta}{\mathrm{d} H^{2}}-\frac{1}{2} \psi^{-\frac{3}{2}} \phi(H) \eta=0 \quad \text { for } H>0,
$$

where $(\psi, \eta) \in\left\{\left(\psi_{b}, \partial_{b} \psi_{b}\right),\left(\psi_{B}, \partial_{B} \psi_{B}\right)\right\}$. This follows from the $C^{1}$-dependence of $\psi_{B}$ and $\psi_{b}$ on $B$ and $b$ for $H \gg_{B} 1$ and $H \ll_{b} 1$, respectively (cf. Proposition 3.1 and Proposition 3.2) together with standard ODE theory in the bulk.

For equation (33) we can prove the following uniqueness result:

Lemma 3.3. Suppose that $\psi:[0, \infty) \rightarrow \mathbb{R}$ is the unique classical solution of problem (15) and $\eta \in C^{0}([0, \infty)) \cap C^{2}((0, \infty))$ is a solution of the corresponding linearized equation (33) fulfilling conditions (32), i.e.

$$
\begin{aligned}
& \eta=0 \quad \text { at } H=0, \\
& \frac{\mathrm{d} \eta}{\mathrm{d} H} \rightarrow 0 \quad \text { as } H \rightarrow \infty .
\end{aligned}
$$

Then $\eta \equiv 0$.

Proof. Consider the function $\eta^{2} \geq 0$ and note that

$$
\frac{1}{2} \frac{\mathrm{d}^{2} \eta^{2}}{\mathrm{~d} H^{2}}=\left(\frac{\mathrm{d} \eta}{\mathrm{d} H}\right)^{2}+\eta \frac{\mathrm{d}^{2} \eta}{\mathrm{d} H^{2}} \stackrel{(33)}{=}\left(\frac{\mathrm{d} \eta}{\mathrm{d} H}\right)^{2}+\frac{1}{2} \psi^{-\frac{3}{2}} \phi(H) \eta^{2} \geq 0 .
$$

Since $\eta^{2} \geq 0$ with $\eta^{2} \stackrel{(34 a)}{=} 0$ at $H=0$, necessarily $\frac{\mathrm{d} \eta^{2}}{\mathrm{~d} H} \geq 0$ for a sequence $H \searrow 0$. By (35), $\frac{\mathrm{d} \eta^{2}}{\mathrm{~d} H}$ is monotonically increasing and therefore $\frac{\mathrm{d} \eta^{2}}{\mathrm{~d} H} \geq 0$ for all $H>0$. Hence

$$
\frac{\mathrm{d}}{\mathrm{d} H}\left(\frac{\mathrm{d} \eta}{\mathrm{d} H}\right)^{2}=2 \frac{\mathrm{d} \eta}{\mathrm{d} H} \frac{\mathrm{d}^{2} \eta}{\mathrm{d} H^{2}} \stackrel{(33)}{=} \frac{1}{2} \psi^{-\frac{3}{2}} \phi(H) \frac{\mathrm{d} \eta^{2}}{\mathrm{~d} H} \geq 0 .
$$

Since $\left(\frac{\mathrm{d} \eta}{\mathrm{d} H}\right)^{2}=0$ at $H=\infty($ cf. $(34 b))$, this implies $\frac{\mathrm{d} \eta}{\mathrm{d} H} \equiv 0$. Again appealing to $(34 a)$, we infer $\eta \equiv 0$.

As a corollary of Lemma 3.3 we obtain: 
Corollary 3.4. Suppose that for each $n \in\left(\frac{3}{2}, \frac{7}{3}\right)$ the parameters $b, B \in \mathbb{R}$ are chosen such that $\psi_{b}=\psi_{B}=: \psi, \psi$ being the unique classical solution of equation (15). Then the vectors

$$
\left(\partial_{b} \psi_{b}, \partial_{H} \partial_{b} \psi_{b}\right) \quad \text { and } \quad\left(\partial_{B} \psi_{B}, \partial_{H} \partial_{B} \psi_{B}\right)
$$

are linearly independent for all $H>0$.

Geometrically this means that the solution manifolds

$$
\left\{\left(H, \psi_{b}, \partial_{H} \psi_{b}\right): b \in \mathbb{R}, H>0\right\}
$$

and

$$
\left\{\left(H, \psi_{B}, \partial_{H} \psi_{B}\right): B \in \mathbb{R}, H>0\right\}
$$

are transversal along $\left(H, \psi, \partial_{H} \psi\right)$.

Proof of Corollary 3.4. The choice of $b$ and $B$ is possible due to Propositions 3.1 and 3.2. By Liouville's formula and standard uniqueness theory of ODEs, the property that the vectors (36) are linearly independent for one $H>0$ is equivalent to the property that they are linearly independent for all $H>0$. Furthermore, by the same arguments the vectors (36) are linearly independent for one $H>0$ if and only if the functions $\partial_{B} \psi_{B}$ and $\partial_{b} \psi_{b}$ are linearly independent. The latter can be easily proven:

Suppose that

$$
\alpha_{B} \partial_{B} \psi_{B}+\alpha_{b} \partial_{b} \psi_{b} \equiv 0 \text { for }\left(\alpha_{B}, \alpha_{b}\right) \in \mathbb{R}^{2} .
$$

Since $\partial_{b} \psi_{b} \not \equiv 0$ (cf. (31)), by Lemma 3.3 and because $\partial_{b} \psi_{b}$ fulfills (34a) (cf. (31c)), $\partial_{b} \psi_{b}$ cannot fulfill (34b). Hence, from (39) and the fact that $\partial_{B} \psi_{B}$ meets $(34 b)$, we infer $\alpha_{b}=0$. Due to $\partial_{B} \psi_{B} \not \equiv 0$ (cf. $(30 c)$ ) necessarily also $\alpha_{B}=0$.

\section{Smoothness in $n$ and conclusion}

Proof of Theorem 2.1. We already know from the uniqueness result in [8] that the solution manifolds (37) and (38) intersect in exactly one curve defining the unique

solution of the dynamical system $\left(H, \frac{\mathrm{d} \psi}{\mathrm{d} H}, \frac{\mathrm{d}^{2} \psi}{\mathrm{d} H^{2}}\right)$ associated to (15). Furthermore, by Propositions 3.1 and 3.2, and standard ODE theory in the bulk, the curves

$$
H \mapsto\left(H, \partial_{b} \psi_{b}, \partial_{H} \partial_{b} \psi_{b}\right) \quad \text { and } \quad H \mapsto\left(H, \partial_{B} \psi_{B}, \partial_{H} \partial_{B} \psi_{B}\right)
$$

locally depend smoothly on $n$. As a consequence, this intersection curve - and thus in particular the parameters $b$ and $B$ - (locally with respect to $H$ ) depends smoothly on $n$. This is a consequence of the transversality given by Corollary 3.4 through the implicit function theorem: The set of equations

$$
\psi_{B}(H)-\psi_{b}(H)=0 \quad \text { and } \quad \partial_{H} \psi_{B}(H)-\partial_{H} \psi_{b}(H)=0,
$$


for any fixed $H>0$, implicitly defines the parameters $B$ and $b$ locally as $C^{1}$-functions of $n$ (cf. Propositions 3.1 and 3.2) provided that

$$
\operatorname{det}\left(\begin{array}{cc}
\partial_{B} \psi_{B}(H) & \partial_{B} \partial_{H} \psi_{B}(H) \\
\partial_{b} \psi_{b}(H) & \partial_{b} \partial_{H} \psi_{b}(H)
\end{array}\right) \neq 0,
$$

the latter following from Corollary 3.4.

\section{Tanner's law}

Here we prove that the unique solution of (15) obtained in [8] indeed satisfies Tanner's law to leading order. We recognize $\phi(H) \stackrel{(15 a)}{=} \frac{2}{3 H^{2}}(1+o(1))$ as $H \rightarrow \infty$ and that problem (15) in this regime is approximately invariant under the scaling $H \rightarrow B H$ for any $B>0$. Capitalizing on this invariance, up to this approximation, the equation becomes autonomous if we introduce the independent variable (cf. (92))

$$
s:=\ln H .
$$

We also introduce the new dependent variable

$$
u:=\psi^{\frac{3}{2}}
$$

as a function of $s$. Then we observe that $(15 a)$ can be recast as

$$
\frac{3}{2} u^{\frac{1}{3}}\left(\frac{\mathrm{d}}{\mathrm{d} s}-1\right) \frac{\mathrm{d}}{\mathrm{d} s} u^{\frac{2}{3}}-1+f=0 \quad \text { for } s \in \mathbb{R},
$$

where

$$
f:=\frac{1}{1+e^{(3-n) s}}
$$

and therefore

$$
\frac{\mathrm{d} v}{\mathrm{~d} s}-v-\frac{v^{2}}{3 u}=-1+f \quad \text { for } s \in \mathbb{R}, \quad \text { with } v:=\frac{\mathrm{d} u}{\mathrm{~d} s} .
$$

We emphasize that the specific form for $f$ in (42) is immaterial for the analysis of this section as long as we have $f=O\left(s^{-2} \ln s\right)$ as $s \rightarrow \infty$. This can be ensured for a variety of models of liquid-solid interaction other than our choice, that is, nonlinear slip with mobility exponent $n \in\left(\frac{3}{2}, \frac{7}{3}\right)$.

Proposition 4.1. The unique classical solution of problem (15) obeys

$$
\left\{\begin{array}{l}
u=s\left(1-\frac{1}{3} s^{-1} \ln s+a s^{-1}+O\left(s^{-2} \ln s\right)\right) \\
v=\frac{\mathrm{d} u}{\mathrm{~d} s}=1-\frac{1}{3} s^{-1}+O\left(s^{-2} \ln s\right) \\
\frac{\mathrm{d}^{2} u}{\mathrm{~d} s^{2}}=O\left(s^{-2} \ln s\right)
\end{array}\right\} \text { as } s \rightarrow \infty
$$

with some $a \in \mathbb{R}$.

Proof. Equation (43) implies

$$
\frac{\mathrm{d} v}{\mathrm{~d} s}-v \geq-1+f
$$


Now we may use $\frac{\mathrm{d} v}{\mathrm{~d} s}-v=e^{s} \frac{\mathrm{d}}{\mathrm{d} s} e^{-s} v$ and integrate the above inequality:

$$
\int_{s}^{\infty} \frac{\mathrm{d}}{\mathrm{d} s^{\prime}}\left(e^{-s^{\prime}} v\left(s^{\prime}\right)\right) \mathrm{d} s^{\prime} \geq-e^{-s}+\int_{s}^{\infty} e^{-s^{\prime}} f\left(s^{\prime}\right) \mathrm{d} s^{\prime} .
$$

In order to evaluate the integral on the left-hand side of (45), we observe that

$$
v \stackrel{(43)}{=} \frac{\mathrm{d} u}{\mathrm{~d} s} \stackrel{(41)}{=} \frac{\mathrm{d}}{\mathrm{d} s} \psi^{\frac{3}{2}} \stackrel{(40)}{=} e^{s} \frac{\mathrm{d}}{\mathrm{d} H} \psi^{\frac{3}{2}}
$$

and therefore

$$
e^{-s} v=\frac{\mathrm{d}}{\mathrm{d} H} \psi^{\frac{3}{2}}=\frac{3}{2} \psi^{\frac{1}{2}} \frac{\mathrm{d} \psi}{\mathrm{d} H} \stackrel{(13),(14 a)}{>} 0 .
$$

We claim $e^{-s} v \rightarrow 0$ as $s \rightarrow \infty$ at least for a subsequence. Else in view of (46), $\liminf _{H \rightarrow \infty} \frac{\mathrm{d}}{\mathrm{d} H} \psi^{\frac{3}{2}}>0$ and therefore, by integration,

$$
\liminf _{H \rightarrow \infty} H^{-\frac{2}{3}} \psi>0
$$

Using equation (15a), we obtain from (47) that $\limsup _{H \rightarrow \infty} H^{\frac{7}{3}}\left|\frac{\mathrm{d}^{2} \psi}{\mathrm{d} H^{2}}\right|<\infty$. By integrating twice, using (15c), we infer that $\psi$ stays bounded as $H \rightarrow \infty$, which contradicts $(47)$.

Therefore (45) yields

$$
0 \stackrel{(46)}{<} v(s)=\frac{\mathrm{d} u}{\mathrm{~d} s}(s) \leq 1-\int_{s}^{\infty} e^{s-s^{\prime}} f\left(s^{\prime}\right) \mathrm{d} s^{\prime} \leq 1 .
$$

On the other hand, using (48) in equation (43), we obtain

$$
\frac{\mathrm{d} v}{\mathrm{~d} s}-v=-1+f+\frac{v^{2}}{3 u} \leq-1+f+\frac{1}{3 u} .
$$

Integrating this equation as before, we conclude

$$
v(s) \geq \int_{s}^{\infty} e^{s-s^{\prime}}\left(1-f\left(s^{\prime}\right)-\frac{1}{3 u\left(s^{\prime}\right)}\right) \mathrm{d} s^{\prime} .
$$

Due to the monotonicity of $u$ stated in (46), we infer from (49)

$$
\frac{\mathrm{d} u}{\mathrm{~d} s}=v \geq 1-f-\frac{1}{3 u} .
$$

From (48) we have $u \leq s(1+o(1))$ as $s \rightarrow \infty$. We know that $u$ is monotonically increasing and $u>0$ by (12). Suppose by contradiction $\lim \sup _{s \rightarrow \infty} u<\infty$. This implies

$$
\limsup _{H \rightarrow \infty} \psi^{(40),(41)} \limsup _{s \rightarrow \infty} u^{\frac{2}{3}}<\infty .
$$

By (13) and (15a) we have that $\limsup _{H \rightarrow \infty} H^{2} \frac{\mathrm{d}^{2} \psi}{\mathrm{d} H^{2}}$ is negative, which, using (15c), implies positivity of $\liminf _{H \rightarrow \infty} H \frac{\mathrm{d} \psi}{\mathrm{d} H}$ and thus $\psi \rightarrow \infty$ as $H \rightarrow \infty$. This is a contradiction to our assumption. 
Therefore $u \rightarrow \infty$ as $s \rightarrow \infty$. By (50) this amounts to $v \geq 1+o(1)$ and $u \geq s(1+o(1))$ as $s \rightarrow \infty$, that is, by (48),

$$
v=1+o(1) \quad \text { and } \quad u=s(1+o(1)) \quad \text { as } s \rightarrow \infty .
$$

Utilizing equation (43) in form of

$$
e^{s} \frac{\mathrm{d}}{\mathrm{d} s} e^{-s} v=\frac{v^{2}}{3 u}-1+f
$$

we infer by integration

$$
v(s)=\int_{s}^{\infty} e^{s-s^{\prime}}\left(1-f\left(s^{\prime}\right)-\frac{\left(v\left(s^{\prime}\right)\right)^{2}}{3 u\left(s^{\prime}\right)}\right) \mathrm{d} s^{\prime} .
$$

Inserting (51) into (52) yields

$$
v=1-\frac{1}{3} s^{-1}+o\left(s^{-1}\right) \quad \text { as } s \rightarrow \infty
$$

and integration gives

$$
u=s\left(1-\frac{1}{3} s^{-1} \ln s+o\left(s^{-1} \ln s\right)\right) \quad \text { as } s \rightarrow \infty .
$$

Once more appealing to (52) and using (53), we obtain the refined asymptotics

$$
\begin{array}{ll}
\frac{\mathrm{d} u}{\mathrm{~d} s}=v=1-\frac{1}{3} s^{-1}+O\left(s^{-2} \ln s\right) & \text { as } s \rightarrow \infty, \\
u=s\left(1-\frac{1}{3} s^{-1} \ln s+a s^{-1}+O\left(s^{-2} \ln s\right)\right) & \text { as } s \rightarrow \infty
\end{array}
$$

with an integration constant $a \in \mathbb{R}$. Finally, from (43) we infer that also

$$
\frac{\mathrm{d}^{2} u}{\mathrm{~d} s^{2}}=O\left(s^{-2} \ln s\right) \quad \text { as } s \rightarrow \infty .
$$

\section{The solution manifold obeying Tanner's law}

For convenience we make another change of variables. Since by $(44) u(s)=s(1+o(1))$ and $v(s)=1+o(1)$ as $s \rightarrow \infty$, we can invert the function $u=u(s)$ for $s \gg 1$. Considering $v=\frac{\mathrm{d} u}{\mathrm{~d} s}$ as a function of $u$, we can rephrase equation (43) as

$$
\begin{array}{ll}
v \frac{\mathrm{d} v}{\mathrm{~d} u}-v-\frac{v^{2}}{3 u}=-1+f & \text { for } u>u_{0}, \\
\frac{\mathrm{d} s}{\mathrm{~d} u}=\frac{1}{v} & \text { for } u>u_{0},
\end{array}
$$

where again $f=\frac{1}{1+e^{(3-n) s}}$ (cf. (42)) and $u_{0}>0$ will be chosen (sufficiently large) later. In view of the asymptotics (44) in Proposition 4.1, we define the new unknowns

$$
w:=v-1+\frac{1}{3 u} \quad \text { and } \quad t:=s-u-\frac{\ln u}{3}+a .
$$


Thus indeed

$$
\lim _{u \rightarrow \infty} u w=0, \quad \lim _{u \rightarrow \infty} u \frac{\mathrm{d} w}{\mathrm{~d} u}=0, \quad \text { and } \quad \lim _{u \rightarrow \infty} t=0
$$

for the unique solution of (15). In terms of $w$ and $t$, the system (54) can be rewritten as

$$
\begin{array}{ll}
\frac{\mathrm{d} w}{\mathrm{~d} u}-w=f+g & \text { for } u>u_{0}, \\
\frac{\mathrm{d} t}{\mathrm{~d} u}=r & \text { for } u>u_{0},
\end{array}
$$

where $g:=g\left(u, w, \frac{\mathrm{d} w}{\mathrm{~d} u}\right)$, where

$$
\begin{aligned}
g & :=g\left(u, w, w^{\prime}\right) \\
& :=-\left(15-\frac{4}{u}\right) \frac{1}{27 u^{2}}+\left(6-\frac{5}{u}\right) \frac{w}{9 u}+\frac{1}{3 u} w^{\prime}+\frac{w^{2}}{3 u}-w w^{\prime}, \\
r & :=r(u, w):=\frac{\frac{1}{9 u^{2}}-\left(1+\frac{1}{3 u}\right) w}{1-\frac{1}{3 u}+w} .
\end{aligned}
$$

In agreement with (56), we assume the boundary conditions $\lim _{u \rightarrow \infty} u w=0$ and $\lim _{u \rightarrow \infty} t=0$. We can then directly read off $\lim _{u \rightarrow \infty} u \frac{\mathrm{d} w}{\mathrm{~d} u}=0$ for any classical solution of (57). In view of the definitions of $f$ and $g$ in (43) and $(57 c),(55)$, and the boundary conditions (56), we have $f+g=O\left(u^{-2}\right)$ as $u \rightarrow \infty$. Then (56)\&(57a) lead to $w(u)=-\int_{u}^{\infty} e^{u-u^{\prime}}(f+g)\left(u^{\prime}\right) \mathrm{d} u^{\prime}=O\left(u^{-2}\right)$ as $u \rightarrow \infty$. Once more appealing to $(57 a)$ and $(57 c)$, we obtain $\frac{\mathrm{d} w}{\mathrm{~d} u}=O\left(u^{-2}\right)$ as $u \rightarrow \infty$. Now appealing to $(57 b)$ and $(57 d)$, we get

$$
w=O\left(u^{-2}\right), \quad \frac{\mathrm{d} w}{\mathrm{~d} u}=O\left(u^{-2}\right), \quad \text { and } \quad t=O\left(u^{-1}\right) \quad \text { as } u \rightarrow \infty .
$$

The advantage of the reformulation (57)\&(58) is that $a$ only appears through $f$ (cf. (42)) and the relation between $t$ and $s$ (cf. (55)) in the problem. As we will prove in the following, the $a$-dependence merely leads to an exponential correction of $v=v(u)$ in $u$. Furthermore, in case of the unperturbed traveling-wave equation (4), respectively (16a), for which $f \equiv 0$, equations $(57 a)$ and $(57 b)$ decouple and $(w, t)$ is independent of $a$.

In view of the boundary conditions (58), we are led to define the following norms:

$$
\begin{aligned}
& \|w\|_{W}:=\max \left\{\sup _{u \geq u_{0}}|u|^{2}|w(u)|, \sup _{u \geq u_{0}}|u|^{2}\left|\frac{\mathrm{d} w}{\mathrm{~d} u}(u)\right|\right\}, \\
& \|t\|_{T}:=\sup _{u \geq u_{0}}|u||t(u)|, \\
& \|(w, t)\|_{W \times T}:=\max \left\{\|w\|_{W}, \varepsilon\|t\|_{T}\right\},
\end{aligned}
$$

where $0<\varepsilon \ll 1$ and $u_{0} \gg 1$ will be conveniently fixed later. Due to (42) and (55), $f$ may be viewed as a local function of $u$ and $t$ and we may therefore define the norm

$$
\|f\|_{F}:=\max _{0 \leq k \leq 2} \sup _{\substack{u \geq u_{0},|t| \leq K\left(\varepsilon u_{0}\right)^{-1}}}|u|^{2-k}\left|\partial_{t}^{k} f(u, t)\right|
$$


with a constant $K>0$ to be fixed later. We note for further reference that

$$
\varepsilon\|t\|_{T} \leq K \Rightarrow|t| \leq K\left(\varepsilon u_{0}\right)^{-1}
$$

The spaces $W, T$, and $F$ are defined as the completion of smooth functions $w=w(u)$, $t=t(u)$, respectively $f=f(u, t)$ with finite norm $\|\cdot\|_{W},\|\cdot\|_{T}$, respectively $\|\cdot\|_{F}$. The norm for $W \times T \times F$ is given by

$$
\|(w, t, f)\|_{W \times T \times F}:=\max \left\{\|w\|_{W}, \varepsilon\|t\|_{T},\|f\|_{F}\right\} .
$$

The following existence and uniqueness result can be obtained:

Proposition 5.1. For $c>0$ we define $N_{F}:=\left\{f \in F:\|f\|_{F}<c\right\}$. Then, provided $K \gg 1, \varepsilon \ll 1, c \ll \varepsilon$, and $u_{0} \gg 1+K$, there exists a $C^{1}$-map $\mathcal{S}: N_{F} \rightarrow W \times T$ with bound $\left\|\partial_{F} \mathcal{S}[f]\right\|_{F \rightarrow W \times T} \lesssim 1$ for $f \in N_{F}$, such that $(w, t):=\mathcal{S}[f]$ solves $(57) \mathcal{E}(58)$.

Proof. We split the proof in several parts:

Reformulation of the problem Let

$$
N:=N_{W \times T} \times N_{F}
$$

with

$$
N_{W \times T}:=\left\{(w, t) \in W \times T:\|(w, t)\|_{W \times T} \leq K\right\}
$$

be a neighborhood of $(w, t, f)=(0,0,0)$. We can rewrite $(57) \&(58)$ as a fixed point

$$
\left(\begin{array}{c}
w \\
t
\end{array}\right)=\mathcal{G}[w, t, f]
$$

where $\mathcal{G}: N \rightarrow W \times T$ is given by

$$
\mathcal{G}[w, t, f]:=\left(\begin{array}{c}
S_{W}\left(f(\cdot, t)+g\left(\cdot, w, \frac{\mathrm{d} w}{\mathrm{~d} u}\right)\right) \\
S_{T} r(\cdot, w)
\end{array}\right)
$$

with integral operators

$$
\begin{aligned}
& S_{W} \phi(u):=-\int_{u}^{\infty} e^{u-u^{\prime}} \phi\left(u^{\prime}\right) \mathrm{d} u^{\prime}, \\
& S_{T} \phi(u):=-\int_{u}^{\infty} \phi\left(u^{\prime}\right) \mathrm{d} u^{\prime} .
\end{aligned}
$$

Note that the asymptotic conditions (58) are implied by the finiteness of the norms (59). Our aim is to apply the contaction-mapping theorem and the implicit function theorem to (64), that is, we need to show that for $0<\varepsilon \ll 1, c \ll \varepsilon, K \gg 1$, and $u_{0} \gg 1+\sqrt{K}$ :

(a) $\mathcal{G} \in C^{1}(N ; W \times T)$; 
Rigorous asymptotics to the thin-film equation and Tanner's law

(b) the derivative $\| \partial_{W \times T} \mathcal{G}[w, t, f]$ has the uniform bound $\uparrow$

$$
\left\|\partial_{W \times T} \mathcal{G}[w, t, f]\right\|_{W \times T \rightarrow W \times T} \leq \frac{1}{2} \quad \text { for every }(w, t, f) \in N
$$

so that in particular $\operatorname{id}_{W \times T}-\partial_{W \times T} \mathcal{G}[w, t, f]: W \times T \rightarrow W \times T$ is for every $(w, t, f) \in N$ an isomorphism of Banach spaces with bound $\left\|\left(\operatorname{id}_{W \times T}-\partial_{W \times T} \mathcal{G}[w, t, f]\right)^{-1}\right\|_{W \times T \rightarrow W \times T} \leq 2$

(c) the map $\mathcal{G}[\cdot, \cdot, f]: N_{W \times T} \rightarrow W \times T$ (where $f \in N_{F}$ is fixed) maps $N$ into itself.

Indeed, by (b) and (c) the contraction-mapping theorem yields a solution map $\mathcal{S}: N_{F} \rightarrow$ $N_{W \times T}$ such that $(w, t):=\mathcal{S}[f]$ solves (64). By (a) and (b) the implicit function theorem implies $\mathcal{S} \in C^{1}\left(N_{F} ; N_{W \times T}\right)$.

We note that formally for the directional derivatives in $W, T$, and $F$, respectively,

$$
\begin{aligned}
& \partial_{W} \mathcal{G}[w, t, f] \partial w \\
& =\left(\begin{array}{c}
S_{W}\left(\frac{\partial g}{\partial w}\left(\cdot, w, \frac{\mathrm{d} w}{\mathrm{~d} u}\right) \partial w+\frac{\partial g}{\partial w^{\prime}}\left(\cdot, w, \frac{\mathrm{d} w}{\mathrm{~d} u}\right) \frac{\mathrm{d} \partial w}{\mathrm{~d} u}\right) \\
S_{T} \frac{\partial r}{\partial w}(\cdot, w) \partial w
\end{array}\right), \\
& \partial_{T} \mathcal{G}[w, t, f] \partial t=\left(\begin{array}{c}
S_{W} \frac{\partial f}{\partial t}(\cdot, t) \partial t \\
0
\end{array}\right), \\
& \partial_{F} \mathcal{G}[w, t, f] \partial f=\left(\begin{array}{c}
S_{W} \partial f \\
0
\end{array}\right) .
\end{aligned}
$$

In particular

$$
\begin{gathered}
\partial_{W \times T} \mathcal{G}[w, t, f]\left(\begin{array}{c}
\partial w \\
\partial t
\end{array}\right) \\
=\left(\begin{array}{c}
S_{W}\left(\frac{\partial g}{\partial w}\left(\cdot, w, \frac{\mathrm{d} w}{\mathrm{~d} u}\right) \partial w+\frac{\partial g}{\partial w^{\prime}}\left(\cdot, w, \frac{\mathrm{d} w}{\mathrm{~d} u}\right) \frac{\mathrm{d} \partial w}{\mathrm{~d} u}+\frac{\partial f}{\partial t}(\cdot, t) \partial t\right) \\
S_{T} \frac{\partial r}{\partial w}(\cdot, w) \partial w
\end{array}\right) .
\end{gathered}
$$

Estimates for $S_{W}$ and $S_{T}$ We start by proving estimates for the integral operators $S_{W}$ and $S_{T}$. We note that for $u \geq u_{0}$

$$
\begin{aligned}
\left|S_{W} \phi(u)\right| & \stackrel{(66 a)}{\leq} \int_{u}^{\infty} e^{u-u^{\prime}}\left|\phi\left(u^{\prime}\right)\right| \mathrm{d} u^{\prime} \\
& \leq \int_{u}^{\infty} e^{u-u^{\prime}}\left(u^{\prime}\right)^{-2} \mathrm{~d} u^{\prime} \times \sup _{u^{\prime} \geq u_{0}}\left|u^{\prime}\right|^{2}\left|\phi\left(u^{\prime}\right)\right| \\
& \leq u^{-2} \sup _{u^{\prime} \geq u_{0}}\left|u^{\prime}\right|^{2}\left|\phi\left(u^{\prime}\right)\right|
\end{aligned}
$$

that is,

$$
\sup _{u \geq u_{0}}|u|^{2}\left|S_{W} \phi(u)\right| \leq \sup _{u \geq u_{0}}|u|^{2}|\phi(u)|
$$

$\|$ Here, $\partial_{W \times T}$ denotes the derivative with respect to $W \times T$.

I The expression $\|\cdot\|_{W \times T \rightarrow W \times T}$ denotes the operator norm of bounded linear operators $W \times T \rightarrow$ $W \times T$. 
Rigorous asymptotics to the thin-film equation and Tanner's law

Since $\frac{\mathrm{d}}{\mathrm{d} u} S_{W} \phi(u)=S_{W} \phi(u)+\phi(u)$, we obtain

$$
\left\|S_{W} \phi\right\|_{W} \leq 2 \sup _{u \geq u_{0}}|u|^{2}|\phi(u)| \text {. }
$$

Similarly

$$
\left|S_{T} \phi(u)\right| \leq \int_{u}^{\infty}\left(u^{\prime}\right)^{-2} \mathrm{~d} u^{\prime} \times \sup _{u^{\prime} \geq u_{0}}\left|u^{\prime}\right|^{2}\left|\phi\left(u^{\prime}\right)\right| \leq u^{-1} \sup _{u^{\prime} \geq u_{0}}\left|u^{\prime}\right|^{2}\left|\phi\left(u^{\prime}\right)\right|
$$

and therefore

$$
\left\|S_{T} \phi\right\|_{T} \leq \sup _{u \geq u_{0}}|u|^{2}|\phi(u)|
$$

$\mathcal{G}[\cdot, \cdot, f]$ is a self-map (proof of $(c)$ ) We can estimate for $(w, t, f) \in N$

$$
\begin{aligned}
& \|\mathcal{G}[w, t, f]\|_{W \times T} \\
& =\max \left\{\left\|S_{W} g\left(\cdot, w, \frac{\mathrm{d} w}{\mathrm{~d} u}\right)\right\|_{W}+\left\|S_{W} f(\cdot, t)\right\|_{W}, \varepsilon\left\|S_{T} r(\cdot, w)\right\|_{T}\right\} \\
& \stackrel{(70)}{\leq} \max \left\{2 \sup _{u \geq u_{0}}|u|^{2}\left|g\left(u, w(u), \frac{\mathrm{d} w}{\mathrm{~d} u}(u)\right)\right|+2 \sup _{u \geq u_{0}}|u|^{2}|f(u, t(u))|,\right. \\
& \left.\quad \sup _{u \geq u_{0}}|u|^{2}|r(u, w(u))|\right\} .
\end{aligned}
$$

Then we have

$$
\begin{aligned}
& \sup _{u \geq u_{0}}|u|^{2}\left|g\left(u, w(u), \frac{\mathrm{d} w}{\mathrm{~d} u}(u)\right)\right| \\
& \stackrel{(57 c),(59 a)}{\lesssim}\left(1+u_{0}^{-1}\right)\left(1+u_{0}^{-1}\|w\|_{W}+u_{0}^{-2}\|w\|_{W}^{2}\right),
\end{aligned}
$$

and

$$
\begin{aligned}
& \sup _{u \geq u_{0}}|u|^{2}|f(u, t(u))| \stackrel{(60),(61)}{\leq}\|f\|_{F}, \\
& \sup _{u \geq u_{0}}|u|^{2}|r(u, w(u))| \stackrel{(57 d),(59 a)}{\lesssim}\left(1-\frac{1}{3 u_{0}}-\frac{\|w\|_{W}}{u_{0}^{2}}\right)^{-1}\left(1+\|w\|_{W}\right) .
\end{aligned}
$$

for $u_{0} \gg 1+\sqrt{\|w\|_{W}}$, i.e. $u_{0} \gg 1+\sqrt{K}$. Due to the definition of $N$ in (63), we obtain

$$
\|\mathcal{G}[w, t, f]\|_{W \times T} \lesssim 1+\frac{K^{2}}{u_{0}^{2}}+\varepsilon(1+K)+c,
$$

which implies that $\mathcal{G}[\cdot, \cdot, f]$ maps $N_{W \times T}$ into itself provided $K \gg 1, u_{0} \gg 1+\sqrt{K}$, $\varepsilon \ll 1$, and $c \ll 1$. 
Rigorous asymptotics to the thin-film equation and Tanner's law

Bound for $\partial_{W \times T} \mathcal{G}[w, t, f]$ (proof of $(b)$ ) We first notice that

$$
\begin{aligned}
\stackrel{\|_{W \times T} \mathcal{G}}{\stackrel{(59),(69)}{\leq}} \max \{t, f](\partial w, \partial t) \|_{W \times T} & \left\{S_{W} \frac{\partial g}{\partial w}\left(\cdot, w, \frac{\mathrm{d} w}{\mathrm{~d} u}\right) \partial w \|_{W}\right. \\
+ & \left\|S_{W} \frac{\partial g}{\partial w^{\prime}}\left(\cdot, w, \frac{\mathrm{d} w}{\mathrm{~d} u}\right) \frac{\mathrm{d} \partial w}{\mathrm{~d} u}\right\|_{W} \\
& \left.+\left\|S_{W} \frac{\partial f}{\partial t}(\cdot, t) \partial t\right\|_{W}, \varepsilon\left\|S_{T} \frac{\partial r}{\partial w}(\cdot, w) \partial w\right\|_{T}\right\} \\
\stackrel{(70)}{\leq} \quad & \max \left\{2 \sup _{u \geq u_{0}}|u|^{2}\left|\frac{\partial g}{\partial w}\left(u, w(u), \frac{\mathrm{d} w}{\mathrm{~d} u}(u)\right) \partial w(u)\right|\right. \\
& +2 \sup _{u \geq u_{0}}|u|^{2}\left|\frac{\partial g}{\partial w^{\prime}}\left(u, w(u), \frac{\mathrm{d} w}{\mathrm{~d} u}(u)\right) \frac{\mathrm{d} \partial w}{\mathrm{~d} u}(u)\right| \\
& \left.+2 \sup _{u \geq u_{0}}|u|^{2}\left|\frac{\partial f}{\partial t}(u, t(u)) \partial t(u)\right|, \varepsilon \sup _{u \geq u_{0}}|u|^{2}\left|\frac{\partial r}{\partial w}(u, w(u)) \partial w(u)\right|\right\} .
\end{aligned}
$$

Then we can estimate separately:

$$
\begin{aligned}
& \sup _{u \geq u_{0}}|u|^{2}\left|\frac{\partial g}{\partial w}\left(u, w(u), \frac{\mathrm{d} w}{\mathrm{~d} u}(u)\right) \partial w(u)\right| \\
& \leq \sup _{u \geq u_{0}}\left|\frac{\partial g}{\partial w}\left(u, w(u), \frac{\mathrm{d} w}{\mathrm{~d} u}(u)\right)\right| \times \sup _{u \geq u_{0}}|u|^{2}|\partial w(u)| \\
& \stackrel{(57 c),(59 a)}{\lesssim}\left(u_{0}^{-1}+u_{0}^{-3}\right)\left(1+\|w\|_{W}\right)\|\partial w\|_{W}, \\
& \sup _{u \geq u_{0}}|u|^{2}\left|\frac{\partial g}{\partial w^{\prime}}\left(u, w(u), \frac{\mathrm{d} w}{\mathrm{~d} u}(u)\right) \frac{\mathrm{d} \partial w}{\mathrm{~d} u}(u)\right| \\
& \lesssim\left(u_{0}^{-1}+u_{0}^{-2}\right)\left(1+\|w\|_{W}\right)\|\partial w\|_{W}, \\
& \sup _{u \geq u_{0}}|u|^{2}\left|\frac{\partial f}{\partial t}(u, t(u)) \partial t(u)\right| \\
& \leq \sup _{u \geq u_{0}}|u|\left|\frac{\partial f}{\partial t}(u, t(u))\right| \times \sup _{u \geq u_{0}}|u||\partial t(u)| \stackrel{(59 b),(60),(61)}{\leq}\|f\|_{F}\|\partial t\|_{T},
\end{aligned}
$$

and, due to $\frac{\partial r}{\partial w} r(u, w)=-\left(1-\frac{1}{3 u}+w\right)^{-2}$,

$$
\begin{aligned}
& \sup _{u \geq u_{0}}|u|^{2}\left|\frac{\partial r}{\partial w}(u, w(u)) \partial w(u)\right| \\
& \leq \sup _{u \geq u_{0}}\left|\frac{\partial r}{\partial w}(u, w(u))\right| \times \sup _{u \geq u_{0}}|u|^{2}|\partial w(u)| \\
& \stackrel{(59 a)}{\lesssim}\left(1-\frac{1}{3 u_{0}}-\frac{\|w\|_{W}}{u_{0}^{2}}\right)^{-2} \times\|\partial w\|_{W}
\end{aligned}
$$


for $u_{0} \gg 1+\sqrt{\|w\|_{W}}$.

Gathering our estimates, we have

$$
\begin{aligned}
& \left\|\partial_{W \times T} \mathcal{G}[w, t, f](\partial w, \partial t)\right\|_{W \times T} \\
& \lesssim \max \left\{u_{0}^{-1}(1+K), \varepsilon^{-1} c, \varepsilon\right\}\|\partial(w, t)\|_{W \times T}
\end{aligned}
$$

for $(w, t, f) \in N$ (cf. (63)) provided $u_{0} \gg 1+\sqrt{K}$. Then we can derive the bound (67) provided $\varepsilon \ll 1, c \ll \varepsilon$, and $u_{0} \gg 1+K$. This implies (b).

Continuous differentiability (proof of $(a)$ ) From the above reasoning, we know that the directional derivatives $\partial_{W} \mathcal{G}[w, t, f]$ and $\partial_{T} \mathcal{G}[w, t, f]$ exist as bounded linear operators $W \rightarrow W \times T$, respectively $T \rightarrow W \times T$ for every $(w, t, f) \in N$. Furthermore,

$$
\begin{aligned}
\left\|\partial_{F} \mathcal{G}[w, t, f] \partial f\right\|_{W \times T} & \stackrel{(68 c)}{=}\left\|S_{W} \partial f\right\|_{W} \\
& \stackrel{(70 a)}{\leq} \sup _{u \geq u_{0}}|u|^{2}|\partial f(u, t(u))| \stackrel{(61)}{\leq}\|\partial f\|_{F}
\end{aligned}
$$

for $(w, t, f) \in N$, that is, also the directional derivative $\partial_{F} \mathcal{F}[w, t, f]$ is a bounded linear operator $F \rightarrow F$ for every $(w, t, f) \in N$. Hence, in order to prove (a), it remains to show continuity of the directional derivatives. Since $\partial_{F} \mathcal{G}[w, t, f] \stackrel{(68 c)}{=} S_{W}$ is independent of $(w, t, f)(\operatorname{cf} .(66 a))$, this statement is trivial for $\partial_{F} \mathcal{G}[w, t, f]$. Hence, we need to show continuity of $\partial_{W \times T} \mathcal{G}=\partial_{W \times T} \mathcal{G}[w, t, f]$ in $N$. In view of the definition of $\partial_{W \times T} \mathcal{G}$ in (69), we apply the triangle inequality as in the previous step and consider four terms separately:

We prove that $S_{W} \frac{\partial g}{\partial w}\left(\cdot, w, \frac{\mathrm{d} w}{\mathrm{~d} u}\right)$ is continuous in $w$ :

$$
\begin{aligned}
& \left\|\left(S_{W} \frac{\partial g}{\partial w}\left(\cdot, w_{1}, \frac{\mathrm{d} w_{1}}{\mathrm{~d} u}\right)-S_{W} \frac{\partial g}{\partial w}\left(\cdot, w_{2}, \frac{\mathrm{d} w_{2}}{\mathrm{~d} u}\right)\right) \partial w\right\|_{W} \\
& \stackrel{(59 a),(70 a)}{\leq} 2 \sup _{u \geq u_{0}}|u|^{2} \mid \frac{\partial g}{\partial w}\left(u, w_{1}(u), \frac{\mathrm{d} w_{1}}{\mathrm{~d} u}(u)\right) \\
& -\frac{\partial g}{\partial w}\left(u, w_{2}(u), \frac{\mathrm{d} w_{2}}{\mathrm{~d} u}(u)\right)|| \partial w(u) \mid \\
& \leq 2 u_{0}^{-2}\left(\sup _{\substack{u \geq u_{0} \\
w \in \operatorname{conv}\left\{w_{1}, w_{2}\right\} \\
w^{\prime} \in \operatorname{conv}\left\{\frac{\mathrm{d} w_{1}}{\mathrm{~d} u}, \frac{\mathrm{d} w_{2}}{\mathrm{~d} u}\right\}}}\left|\frac{\partial^{2} g}{\partial w^{2}}\left(u, w, w^{\prime}\right)\right|\right. \\
& +\sup _{\substack{u \geq u_{0} \\
w \in \operatorname{conv}\left\{w_{1}, w_{2}\right\} \\
w^{\prime} \in \operatorname{conv}\left\{\frac{\mathrm{d} w_{1}}{\mathrm{~d} u}, \frac{\mathrm{d} w_{2}}{\mathrm{~d} u}\right\}}}\left|\frac{\partial^{2} g}{\partial w \partial w^{\prime}}\left(u, w, w^{\prime}\right)\right| \mid \\
& \times\left\|w_{1}-w_{2}\right\|_{W}\|\partial w\|_{W}
\end{aligned}
$$


Rigorous asymptotics to the thin-film equation and Tanner's law

$\stackrel{(57 c)}{\lesssim} \quad u_{0}^{-2}\left(1+u_{0}^{-1}\right)\left\|w_{1}-w_{2}\right\|_{W}\|\partial w\|_{W}$

and by a completely analogous reasoning

$$
\begin{aligned}
& \left\|\left(S_{W} \frac{\partial g}{\partial w^{\prime}}\left(\cdot, w_{1}, \frac{\mathrm{d} w_{1}}{\mathrm{~d} u}\right)-S_{W} \frac{\partial g}{\partial w^{\prime}}\left(\cdot, w_{2}, \frac{\mathrm{d} w_{2}}{\mathrm{~d} u}\right)\right) \partial w\right\|_{W} \\
& \lesssim u_{0}^{-2}\left\|w_{1}-w_{2}\right\|_{W}\|\partial w\|_{W},
\end{aligned}
$$

showing continuity of $S_{W} \frac{\partial g}{\partial w^{\prime}}\left(\cdot, w, \frac{\mathrm{d} w}{\mathrm{~d} u}\right)$ in $w$.

Next we show continuity of $S_{W} \frac{\partial f}{\partial t}(\cdot, t)$ in $t$ :

$$
\begin{aligned}
& \left\|\left(S_{W} \frac{\partial f}{\partial t}\left(\cdot, t_{1}\right)-S_{W} \frac{\partial f}{\partial t}\left(\cdot, t_{2}\right)\right) \partial t\right\|_{W} \\
& \stackrel{(70 a)}{\leq} \quad 2 \sup _{u \geq u_{0}}|u|^{2}\left|\frac{\partial f}{\partial t}\left(u, t_{1}(u)\right)-\frac{\partial f}{\partial t}\left(u, t_{2}(u)\right)\right||\partial t(u)| \\
& \stackrel{(59 b)}{\leq} 2 \sup _{\substack{u \geq u_{0} \\
t \in \operatorname{conv}\left\{t_{1}, t_{2}\right\}}}\left|\frac{\partial^{2} f}{\partial t^{2}}(u, t)\right| \times\left\|t_{1}-t_{2}\right\|_{T}\|\partial t\|_{T} \\
& \stackrel{(60),(61)}{\leq} 2\|f\|_{F}\left\|t_{1}-t_{2}\right\|_{T}\|\partial t\|_{T} .
\end{aligned}
$$

Finally, continuity of $S_{T} \frac{\partial r}{\partial w}(\cdot, w)$ in $w$ follows from:

$$
\begin{aligned}
& \left\|\left(S_{T} \frac{\partial r}{\partial w}\left(\cdot, w_{1}\right)-S_{T} \frac{\partial r}{\partial w}\left(\cdot, w_{2}\right)\right) \partial w\right\|_{T} \\
& \stackrel{(70 b)}{\leq} \sup _{u \geq u_{0}}|u|^{2}\left|\frac{\partial r}{\partial w}\left(u, w_{1}(u)\right)-\frac{\partial r}{\partial w}\left(u, w_{2}(u)\right)\right||\partial w(u)| \\
& \stackrel{(59 a)}{\leq} u_{0}^{-2} \sup _{\substack{u \geq u_{0} \\
w \in \operatorname{conv}\left\{w_{1}, w_{2}\right\}}}\left|\frac{\partial^{2} r}{\partial w^{2}}(u, w)\right| \times\left\|w_{1}-w_{2}\right\|_{W}\|\partial w\|_{W} \\
& \stackrel{(57 d)}{\lesssim} u_{0}^{-2}\left(1-\frac{1}{3 u_{0}}-\frac{K}{u_{0}^{2}}\right)^{-3}\left\|w_{1}-w_{2}\right\|_{W}\|\partial w\|_{W},
\end{aligned}
$$

using $(w, t, f) \in N\left(\right.$ cf. (63)) and $u_{0} \gg 1+\sqrt{K}$.

Bound for the solution map $\mathcal{S}$ For deriving the bound on $\mathcal{S}$, we differentiate (64) with respect to $f$ and obtain

$$
\partial_{F} \mathcal{S}[f]=-\left(\operatorname{id}_{W \times T}-\partial_{W \times T} \mathcal{G}[\mathcal{S}[f], f]\right)^{-1} \partial_{F} \mathcal{G}[\mathcal{S}[f], f] .
$$

Thus the claim follows from

$$
\begin{aligned}
& \left\|\partial_{F} \mathcal{S}[f]\right\|_{F \rightarrow W \times T} \\
& \leq\left\|\left(\operatorname{id}_{W \times T}-\partial_{W \times T} \mathcal{G}[\mathcal{S}[f], f]\right)^{-1}\right\|_{W \times T \rightarrow W \times T}\left\|\partial_{F} \mathcal{G}[\mathcal{S}[f], f]\right\|_{F \rightarrow W \times T} \\
& \stackrel{(71)}{\leq}\left\|\left(\operatorname{id}_{W \times T}-\partial_{W \times T} \mathcal{G}[\mathcal{S}[f], f]\right)^{-1}\right\|_{W \times T \rightarrow W \times T} \leq 2
\end{aligned}
$$

by $(b)$. 
From now on, we universally fix $\varepsilon, c$, and $K$ as in Proposition 5.1.

Corollary 5.2. For any $a \in \mathbb{R}$ and $f_{a}$ given by (42) and (55), i.e.

$$
f_{a}(u, t)=\frac{1}{1+u^{\frac{3-n}{3}} e^{(3-n)(u+t-a)}},
$$

(57)\&(58) admits a unique classical solution $(w, t)=\left(w_{a}, t_{a}\right)$ with $\left\|\left(w_{a}, t_{a}\right)\right\|_{W \times T} \lesssim 1$ for $u_{0} \gg 1+a_{+}$. Furthermore, for $f \equiv 0$, (57)E(58) admits a unique classical solution $(w, t)=\left(w_{\mathrm{T}}, t_{\mathrm{T}}\right)$ with $\left\|\left(w_{\mathrm{T}}, t_{\mathrm{T}}\right)\right\|_{W \times T} \lesssim 1$ for $u_{0} \gg 1$. The difference obeys

$$
\left\|\left(w_{a}, t_{a}\right)-\left(w_{\mathrm{T}}, t_{\mathrm{T}}\right)\right\|_{W \times T} \lesssim\left\|f_{a}\right\|_{F} \lesssim u_{0}^{\frac{3+n}{3}} e^{-(3-n)\left(u_{0}-a\right)}
$$

for $u_{0} \gg 1+a_{+}$. The solution $\left(w_{a}, t_{a}\right)$ has a $C^{1}$-dependence on a and $n$ with the asymptotic bound

$$
\left\|\left(\partial_{a} w_{a}, \partial_{a} t_{a}\right)\right\|_{W \times T} \lesssim u_{0}^{\frac{3+n}{3}} e^{-(3-n)\left(u_{0}-a\right)} \quad \text { for } u_{0} \gg 1+a_{+} .
$$

Due to Proposition 4.1, the unique classical solution of problem (15) coincides with the one constructed in Corollary 5.2 if the value for $a$ is the same.

Proof. Since for $f \equiv 0$ trivially $f \in N_{F}$ (where $N_{F}$ is defined as in Proposition 5.1), the construction of $\left(w_{\mathrm{T}}, t_{\mathrm{T}}\right)$ immediately follows by applying Proposition 5.1.

For the construction of $\left(w_{a}, t_{a}\right)$ it remains to show that $\|f\|_{F} \ll 1$ for $u_{0} \gg 1+a_{+}$. The derivatives of $f_{a}$ can be computed to be

$$
\frac{\partial f_{a}}{\partial t}(u, t)=-\frac{(3-n) u^{\frac{3-n}{3}} e^{(3-n)(u+t-a)}}{\left(1+u^{\frac{3-n}{3}} e^{(3-n)(u+t-a)}\right)^{2}}
$$

and

$$
\frac{\partial^{2} f_{a}}{\partial t^{2}}(u, t)=-\frac{\left(1-u^{\frac{3-n}{3}} e^{(3-n)(u+t-a)}\right)(3-n)^{2} u^{\frac{3-n}{3}} e^{(3-n)(u+t-a)}}{\left(1+u^{\frac{3-n}{3}} e^{(3-n)(u+t-a)}\right)^{3}}
$$

Estimates in the $F$-norm are confined to $|t| \lesssim u_{0}^{-1}$ (cf. (60)). There, we have $u_{0}+t-a=u_{0}\left(1+\frac{t-a}{u_{0}}\right) \gtrsim u_{0}$ provided that $u_{0} \gg 1+a_{+}$. Then for $u_{0} \gg 1+a_{+}$indeed

$$
u^{\frac{3-n}{3}} e^{(3-n)(u+t-a)} \gtrsim u_{0}^{\frac{3-n}{3}} e^{(3-n) u_{0}} \gg 1
$$

and we have

$$
\left\|f_{a}\right\|_{F} \lesssim u_{0}^{\frac{3+n}{3}} e^{-(3-n) u_{0}} \ll 1 \quad \text { for } u_{0} \gg 1+a_{+} .
$$

Since $\left(w_{a}, t_{a}\right):=\mathcal{S}\left[f_{a}\right]$, where $\mathcal{S}$ is the $C^{1}$-solution map $N_{F} \rightarrow W \times T$ of Proposition 5.1, and $f_{a}$ is a $C^{1}$-function of $a$ and $n$ into $F$ (because of

$$
\partial_{a} f_{a}=\frac{(3-n) u^{\frac{3-n}{3}} e^{(3-n)(u+t-a)}}{\left(1+u^{\frac{3-n}{3}} e^{(3-n)(u+t-a)}\right)^{2}}
$$


and analogous expressions for derivatives in $t$ ), also $w_{a}$ and $t_{a}$ are $C^{1}$-functions of $a$ and $n$. Explicitly we have

$$
\left(\partial_{a} w_{a}, \partial_{a} t_{a}\right)=\partial_{F} \mathcal{S}\left[f_{a}\right] \partial_{a} f_{a} \quad \text { so that } \quad\left\|\left(\partial_{a} w_{a}, \partial_{a} t_{a}\right)\right\|_{W \times T} \lesssim\left\|\partial_{a} f_{a}\right\|_{F}
$$

by uniform boundedness of the derivative $\partial_{F} \mathcal{S}\left[f_{a}\right]: F \rightarrow W \times T$. Due to (75) and similar expressions for derivatives in $t,\left\|\partial_{a} f_{a}\right\|_{F} \lesssim u_{0}^{\frac{3+n}{3}} e^{-(3-n)\left(u_{0}-a\right)}$.

Finally, the difference formula (72) can be proven using the Lipschitz bound on $\mathcal{S}$, that is, $\left\|\left(w_{a}, t_{a}\right)-\left(w_{\mathrm{T}}, t_{\mathrm{T}}\right)\right\|_{W \times T} \lesssim\left\|f_{a}\right\|_{F}$, so that (72) follows from (74).

It remains to translate these results into corresponding results for $\psi$. This can be done in two steps:

Lemma 5.3. Let $a \in \mathbb{R}$ and denote by $u_{a}=u_{a}(s)$ the inverse function of $s_{a}=s_{a}(u)=$ $u+\frac{\ln u}{3}-a+t_{a}(u)(c f .(55))$, where $\left(w_{a}, t_{a}\right)$ is the unique solution of (57)\&(58) with $f$ as in (42) (cf. Corollary 5.2). Furthermore, define $u_{\mathrm{T}}=u_{\mathrm{T}}(s)$ as the inverse function of $s_{\mathrm{T}}=s_{\mathrm{T}}(u)=u+\frac{\ln u}{3}+t_{\mathrm{T}}(u)$ (cf. (55)), where $\left(w_{\mathrm{T}}, t_{\mathrm{T}}\right)$ is the unique solution of (57)\&(58) with $f \equiv 0$ (cf. Corollary 5.2). Then $u_{a}=u_{a}(s)$ and $u_{\mathrm{T}}=u_{\mathrm{T}}($ s) are welldefined for $s \gg 1+a_{-}$, resp. $s \gg 1$, with

$$
\max _{k=0,1,2}\left|\frac{\mathrm{d}^{k} u_{a}}{\mathrm{~d} s^{k}}(s)-\frac{\mathrm{d}^{k} u_{\mathrm{T}}}{\mathrm{d} s^{k}}(s+a)\right| \lesssim e^{-(3-n)(s+a)} \quad \text { for } s \gg 1+a_{-},
$$

where

$$
\left|u_{a}(s)-\left(s-\frac{\ln s}{3}+a\right)\right| \lesssim s^{-1} \quad \text { and } \quad\left|u_{\mathrm{T}}(s)-\left(s-\frac{\ln s}{3}\right)\right| \lesssim s^{-1}
$$

for $s \gg 1+a_{-}$. The function $u_{a}(s)$ is locally in $s$ a $C^{1}$-function of a and $n$ with the asymptotic expression

$$
\partial_{a} u_{a}=-1+o(1) \quad \text { as } s \rightarrow \infty .
$$

Proof. First we note that due to (55) and since

$$
\left\|t_{a}\right\|_{T} \lesssim 1 \text { and }\left\|t_{T}\right\|_{T} \lesssim 1
$$

by Corollary 5.2 , the functions $s_{a}=s_{a}(u)$ and $s_{\mathrm{T}}=s_{\mathrm{T}}(u)$ are strictly monotone and therefore the inverse functions $u_{a}=u_{a}(s)$ and $u_{\mathrm{T}}=u_{\mathrm{T}}(s)$ are well-defined and $C^{1}$ functions of $a$ and $n$ for $s \gg 1+a_{+}-a=1+a_{-}$. The asymptotic expansion (77) immediately follows from the definition of $u_{\mathrm{T}},(55)$, and (79). It follows from (77) that

$$
u_{a}(s)-u_{\mathrm{T}}(s+a)=o(1) \quad \text { as } s \rightarrow \infty .
$$

We define

$$
v_{a}:=w_{a}+1-\frac{1}{3 u} \quad \text { and } \quad v_{\mathrm{T}}:=w_{\mathrm{T}}+1-\frac{1}{3 u} .
$$

By reversing the computations in (43) and (54)-(57), we see that

$$
\frac{\mathrm{d} u_{a}(s)}{\mathrm{d} s}=v_{a}\left(u_{a}(s)\right)
$$


Rigorous asymptotics to the thin-film equation and Tanner's law

Therefore

$$
\begin{aligned}
\frac{\mathrm{d} u_{a}}{\mathrm{~d} s}(s)-\frac{\mathrm{d} u_{\mathrm{T}}}{\mathrm{d} s}(s+a) \stackrel{(82)}{=} & v_{a}\left(u_{a}(s)\right)-v_{\mathrm{T}}\left(u_{\mathrm{T}}(s+a)\right) \\
= & \left(v_{a}\left(u_{a}(s)\right)-v_{\mathrm{T}}\left(u_{a}(s)\right)\right) \\
& +\left(v_{\mathrm{T}}\left(u_{a}(s)\right)-v_{\mathrm{T}}\left(u_{\mathrm{T}}(s+a)\right)\right) .
\end{aligned}
$$

By estimate (72) of Corollary 5.2 we have

$$
\begin{aligned}
\left|v_{a}\left(u_{a}(s)\right)-v_{\mathrm{T}}\left(u_{a}(s)\right)\right| & \stackrel{(81)}{=}\left|w_{a}\left(u_{a}(s)\right)-w_{\mathrm{T}}\left(u_{a}(s)\right)\right| \\
& \stackrel{(59),(72)}{\lesssim}\left(u_{a}(s)\right)^{-\frac{3-n}{3}} e^{-(3-n) u_{a}(s)} \\
& \lesssim e^{-(3-n)(s+a)} \quad \text { for } s \gg 1+a_{-} .
\end{aligned}
$$

Furthermore, by the mean value theorem,

$$
\begin{aligned}
& \left|v_{\mathrm{T}}\left(u_{a}(s)\right)-v_{\mathrm{T}}\left(u_{\mathrm{T}}(s+a)\right)\right| \\
& \lesssim \max _{\sigma \in[0,1]}\left|\frac{\mathrm{d} v_{\mathrm{T}}}{\mathrm{d} u}\left(\sigma u_{a}(s)+(1-\sigma) u_{\mathrm{T}}(s+a)\right)\right| \times\left|u_{a}(s)-u_{\mathrm{T}}(s+a)\right| .
\end{aligned}
$$

Since by (81) $\frac{\mathrm{d} v_{\mathrm{T}}}{\mathrm{d} u}=\frac{\mathrm{d} w_{\mathrm{T}}}{\mathrm{d} u}-\frac{1}{3 u^{2}}$ and since $\left\|w_{\mathrm{T}}\right\|_{W} \lesssim 1$ for $s \gg 1+a_{-}$, we obtain

$$
\begin{aligned}
& \left|v_{\mathrm{T}}\left(u_{a}(s)\right)-v_{\mathrm{T}}\left(u_{\mathrm{T}}(s+a)\right)\right| \\
& \lesssim\left(\left(u_{a}(s)\right)^{-2}+\left(u_{\mathrm{T}}(s+a)\right)^{-2}\right)\left|u_{a}(s)-u_{\mathrm{T}}(s+a)\right| \\
& \stackrel{(77)}{\lesssim} s^{-2}\left|u_{a}(s)-u_{\mathrm{T}}(s+a)\right| \quad \text { for } s \gg 1+a_{-} .
\end{aligned}
$$

Therefore (83) turns into

$$
\left|\frac{\mathrm{d} u_{a}}{\mathrm{~d} s}(s)-\frac{\mathrm{d} u_{\mathrm{T}}}{\mathrm{d} s}(s+a)\right| \lesssim e^{-(3-n)(s+a)}+s^{-2}\left|u_{a}(s)-u_{\mathrm{T}}(s+a)\right|
$$

for $s \gg 1+a_{-}$. Integrating from $s=\infty$ and using (80), we get

$$
\sup _{s \geq s_{0}}\left|u_{a}(s)-u_{\mathrm{T}}(s+a)\right| \lesssim e^{-(3-n)\left(s_{0}+a\right)}+s_{0}^{-1} \sup _{s \geq s_{0}}\left|u_{a}(s)-u_{\mathrm{T}}(s+a)\right|
$$

for $s_{0} \gg 1+a_{-}$, implying (76) for $k=0$. (76) for $k=1$ follows from (84) and the case $k=2$ can be obtained using equation (43).

Finally, estimate (73) of Corollary 5.2 implies $\partial_{a} t_{a}=o(1)$ as $u \rightarrow \infty$ and therefore

$$
\partial_{a} s_{a}(u) \stackrel{(55)}{=}-1+o(1) \quad \text { as } u \rightarrow \infty \text {. }
$$

Differentiating $u_{a}\left(s_{a}(u)\right) \equiv u$ with respect to $a$, we obtain

$$
\partial_{a} u_{a}=-\left(\partial_{a} s_{a}\right)\left(\partial_{s} u_{a}\right) \stackrel{(82)}{=}-v_{a}\left(\partial_{a} s_{a}\right) \stackrel{(85)}{=} v_{a}(1+o(1)) \quad \text { as } u \rightarrow+\infty \text {; }
$$

since $\left\|w_{a}\right\|_{W} \lesssim 1$, it follows from $(81)$ that $v_{a}(u)=1+o(1)$ as $u \rightarrow \infty$, whence (78).

For any $B=e^{a}>0$, we are able to characterize a solution $\psi=\psi_{B}$ to $(15 a) \&(15 c)$ and to characterize the leading-order asymptotics as $H \rightarrow \infty$ : 
Proof of Proposition 3.1. For given $B>0$, let $u_{a}=u_{a}(s)$ be defined as in Lemma 5.3 with $a=\ln B$ and let $s=\log H$. Then $\psi_{B}=\psi_{B}(H)$, defined by $\psi_{B}(H):=\left(u_{a}(\ln H)\right)^{\frac{2}{3}}$ (cf. (40)\&(41)), solves $(15 a) \&(15 c)$ for $H \gg 1+B^{-1}$. In the same way we define $\psi_{\mathrm{T}}(H):=\left(u_{\mathrm{T}}(\ln H)\right)^{\frac{2}{3}}$ (where $u_{\mathrm{T}}$ is defined as in Lemma 5.3), being a solution of (16) for $H \gg 1$. The asymptotic expansion (18) immediately follows from (77) (cf. Lemma 5.3). Also the regularity in $B$ and $n$ is immediate from the respective statements for $u_{a}$ in Lemma 5.3 and standard ODE theory in the bulk.

The comparison formula (30a)\&(30b) follows from transformation (41):

$$
\psi_{B}-\psi_{\mathrm{T}}=u_{a}^{\frac{2}{3}}-u_{\mathrm{T}}^{\frac{2}{3}}=\frac{\left(u_{a}-u_{\mathrm{T}}\right)\left(u_{a}^{\frac{1}{3}}+u_{\mathrm{T}}^{\frac{1}{3}}\right)}{u_{a}^{\frac{2}{3}}+u_{a}^{\frac{1}{3}} u_{\mathrm{T}}^{\frac{1}{3}}+u_{\mathrm{T}}^{\frac{2}{3}}} .
$$

By (76) and (77), we can infer

$$
\left|\frac{\psi_{B}-\psi_{\mathrm{T}}}{\psi_{\mathrm{T}}}\right| \lesssim B^{3-n} s^{-1} e^{-(3-n) s} \quad \text { for } s \gg 1+(\ln B)_{-},
$$

which leaves us with (30b) using $s=\ln H$ (cf. (40)).

It remains to prove the asymptotic expression $(30 c)$. We notice that

$$
\partial_{B} \psi_{B}=\left(\partial_{a} u_{a}^{\frac{2}{3}}\right)\left(\partial_{B} a\right)=-\frac{2}{3 B} u_{a}^{-\frac{1}{3}} \partial_{a} u_{a} \stackrel{(77),(78)}{=} \frac{2}{3 B}(\ln H)^{-\frac{1}{3}}(1+o(1))
$$

as $H \rightarrow \infty$. Differentiating $(15 a)$ yields

$$
\partial_{H}^{2} \partial_{B} \psi_{B}=\frac{1}{2} \psi_{B}^{-\frac{3}{2}} \phi(H) \partial_{B} \psi_{B} \stackrel{(77),(86)}{=} \frac{2}{9 B}(\ln H)^{-\frac{4}{3}} H^{-2}(1+o(1))
$$

as $H \rightarrow \infty$, so that integration immediately implies $(30 c)$.

\section{The asymptotics near the contact line}

First we prove that indeed $\psi(H)=\psi_{\mathrm{TW}}(H)(1+o(1))$ as $H \searrow 0$, where $\psi$ is a solution of $(15 a) \&(15 b)$ and $\psi_{\mathrm{TW}}$ is given by $(21)$ :

Lemma 6.1. Let $n \in\left(\frac{3}{2}, 3\right)$. Any classical solution $\psi$ of problem (15a)G(15b) fulfills the asymptotics

$$
\begin{array}{ll}
\psi(H)=C H^{2-\frac{2}{3} n}(1+o(1)) & \text { as } H \searrow 0, \\
\frac{\mathrm{d} \psi}{\mathrm{d} H}(H)=C\left(2-\frac{2}{3} n\right) H^{1-\frac{2}{3} n}(1+o(1)) & \text { as } H \searrow 0, \\
\frac{\mathrm{d}^{2} \psi}{\mathrm{d} H^{2}}(H)=C\left(2-\frac{2}{3} n\right)\left(1-\frac{2}{3} n\right) H^{-\frac{2}{3} n}(1+o(1)) & \text { as } H \searrow 0,
\end{array}
$$

where $C$ is defined in (21).

Proof. The proof is inspired by - and simplifies - the arguments by Taliaferro in [38, Section 3]. Let $\psi_{\mathrm{TW}}$ be defined by (21). We claim

$$
\lim _{H \rightarrow 0^{+}} \frac{\psi(H)}{\psi_{T W}(H)}=L \in[0,+\infty]
$$


and

$$
\lim _{H \rightarrow 0^{+}} \frac{\mathrm{d} \psi_{T W}}{\mathrm{~d} H}(H)=+\infty, \quad \lim _{H \rightarrow 0^{+}} \frac{\mathrm{d} \psi(H)}{\mathrm{d} H}=+\infty .
$$

Assuming (88) and (89), we have

$$
\begin{aligned}
L & =\lim _{H \rightarrow 0^{+}} \frac{\psi(H)}{\psi_{\mathrm{TW}}(H)}=\lim _{H \rightarrow 0^{+}} \frac{\frac{\mathrm{d} \psi(H)}{\mathrm{d} H}}{\frac{\mathrm{d} \psi_{\mathrm{TW}}(H)}{\mathrm{d} H}}=\lim _{H \rightarrow 0^{+}} \frac{\frac{\mathrm{d}^{2} \psi(H)}{\mathrm{d} H^{2}}}{\frac{\mathrm{d}^{2} \psi_{\mathrm{TW}}(H)}{\mathrm{d} H^{2}}} \\
& =\lim _{H \rightarrow 0^{+}} \frac{H^{n-1} \sqrt{\psi_{\mathrm{TW}}(H)}}{\left(H^{2}+H^{n-1}\right) \sqrt{\psi(H)}}=\frac{1}{\sqrt{L}},
\end{aligned}
$$

hence $L=1$ and (87) follow from (90).

In order to prove (88), we consider the function $H(\sigma), \sigma \in\left(0, \sigma_{0}\right)$, implicitly defined through

$$
\begin{aligned}
& \sigma=: \int_{H(\sigma)}^{1} \frac{\mathrm{d} \tilde{H}}{\left(\psi_{\mathrm{TW}}(\tilde{H})\right)^{2}} \nearrow \sigma_{0}:=\int_{0}^{1} \frac{\mathrm{d} \tilde{H}}{\left(\psi_{\mathrm{TW}}(\tilde{H})\right)^{2}} \in(0, \infty], \\
& \mu(\sigma):=\frac{\psi(H(\sigma))}{\psi_{T W}(H(\sigma))}-1 .
\end{aligned}
$$

After straightforward computations using (15a) and (21), we find

$$
\frac{\mathrm{d}^{2} \mu}{\mathrm{d} \sigma^{2}}=\frac{\left(\Psi_{\mathrm{TW}}(H)\right)^{\frac{5}{2}}}{H^{n-1}}\left(1+\mu-\frac{1}{1+H^{3-n}} \frac{1}{\sqrt{1+\mu}}\right) .
$$

Assume by contradiction that (88) is false. Then sequences $\sigma_{k}^{\prime} \nearrow \sigma_{0}$ and $\sigma_{k}^{\prime \prime} \nearrow \sigma_{0}$ of local maxima and minima of $\mu$, respectively, exist such that $\mu\left(\sigma_{k}^{\prime}\right) \rightarrow L^{\prime}>L^{\prime \prime} \leftarrow \mu\left(\sigma_{k}^{\prime \prime}\right)$ as $k \rightarrow+\infty$. In particular, we have $\frac{\mathrm{d}^{2} \mu}{\mathrm{d} \sigma^{2}}\left(\sigma_{k}^{\prime}\right) \leq 0 \leq \frac{\mathrm{d}^{2} \mu}{\mathrm{d} \sigma^{2}}\left(\sigma_{k}^{\prime \prime}\right)$ and thus by (91)

$$
\left(1+\mu\left(\sigma_{k}^{\prime}\right)\right)^{\frac{3}{2}} \leq \frac{1}{1+H\left(\sigma_{k}^{\prime}\right)^{3-n}} \quad \text { and } \quad\left(1+\mu\left(\sigma_{k}^{\prime \prime}\right)\right)^{\frac{3}{2}} \geq \frac{1}{1+H\left(\sigma_{k}^{\prime \prime}\right)^{3-n}} .
$$

Since $H\left(\sigma_{k}^{\prime}\right) \rightarrow 0$ and $H\left(\sigma_{k}^{\prime \prime}\right) \rightarrow 0$ as $k \rightarrow+\infty$, this implies $L^{\prime} \leq 0 \leq L^{\prime \prime}$, a contradiction. Therefore (88) holds.

Since the first part of (89) is obvious, it remains to show the second part. First of all, the limit $L^{\prime}=\lim _{H \rightarrow 0} \frac{\mathrm{d} \psi}{\mathrm{d} H}$ exists (since $\frac{\mathrm{d}^{2} \psi}{\mathrm{d} H^{2}}$ is negative) and is nonnegative (since $\psi$ is positive for $H>0$ with $\psi(0)=0$ ). If $L^{\prime}<+\infty$, we would have $\psi(H)<\left(1+L^{\prime}\right) H$ as $H \rightarrow 0$, hence $\frac{\mathrm{d}^{2} \psi}{\mathrm{d} H^{2}} \stackrel{(15 a)}{\lesssim}-\frac{1}{H^{n-1 / 2}}$ as $H \rightarrow 0$ : since $n>3 / 2$, this contradicts $L^{\prime}<+\infty$. Hence $L^{\prime}=+\infty$.

In order to parametrize solutions to problem $(15 a) \&(15 b)$ and to describe their dependence on $n$, we now perform a sequence of transformations that reduces the problem to the study of invariant manifolds of a suitable dynamical system. 
Rigorous asymptotics to the thin-film equation and Tanner's law

Coordinate transformations

We use the coordinate transformation

$$
s:=\ln H,
$$

so that the contact line is shifted to $s=-\infty$. Motivated by the leading-order behavior (87), we introduce the new unknown

$$
1+\mu:=\frac{\psi}{\psi_{\mathrm{TW}}}=C^{-1} e^{-\left(2-\frac{2}{3} n\right) s} \psi .
$$

Hence, using the commutation relation $\frac{\mathrm{d}}{\mathrm{d} s} e^{\varphi s}=e^{\varphi s}\left(\frac{\mathrm{d}}{\mathrm{d} s}+\varphi\right)$ for $\varphi \in \mathbb{R}$, problem $(15 a) \&(15 b)$ turns into

$$
\begin{aligned}
& (1+\mu)^{\frac{1}{2}}\left(3 \frac{\mathrm{d}}{\mathrm{d} s}-(2 n-3)\right)\left(3 \frac{\mathrm{d}}{\mathrm{d} s}+2(3-n)\right)(1+\mu) \\
& =-\frac{2(3-n)(2 n-3)}{1+e^{(3-n) s}} \quad \text { for } s \in \mathbb{R}
\end{aligned}
$$

and

$$
\lim _{s \rightarrow-\infty} \mu=0
$$

\section{Reformulation as a dynamical system}

We reformulate (94a) as an autonomous three-dimensional continuous dynamical system by introducing the additional functions

$$
r:=e^{(3-n) s} \quad \text { and } \quad p:=\frac{\mathrm{d} \mu}{\mathrm{d} s} .
$$

Thus (94a) turns into

$$
\frac{\mathrm{d}}{\mathrm{d} s}\left(\begin{array}{c}
r \\
\mu \\
p
\end{array}\right)=F(r, \mu, p) \quad \text { for }-s \gg 1,
$$

where

$$
\begin{aligned}
F(r, \mu, p):= & \left(\begin{array}{c}
(3-n) r \\
p \\
F_{3}(r, \mu, p)
\end{array}\right), \\
9 F_{3}(r, \mu, p):= & 3(4 n-9) p+2(2 n-3)(3-n) v \\
& +2(3-n)(2 n-3)\left(1-\frac{(1+\mu)^{-\frac{1}{2}}}{1+r}\right) .
\end{aligned}
$$

As desired, we have

$$
F(0,0,0)=(0,0,0)
$$

i.e. $(0,0,0)$ is a stationary point of $(95)$. The unique solution to (15) converges to it as $s \rightarrow-\infty$ : 
Lemma 6.2. We have $(r, \mu, p) \rightarrow(0,0,0)$ as $s \rightarrow-\infty$ for the unique solution of problem (15).

Proof. We note that, utilizing (87a) and the transformations (92) and (93), indeed $\mu \rightarrow 0$ as $s \rightarrow-\infty$ for the unique solution of (15). Trivially $r \rightarrow 0$ as $s \rightarrow-\infty$. For $p$ we may differentiate $(93)$ and obtain $p=C^{-1} e^{-\left(2-\frac{2}{3} n\right) s}\left(\frac{\mathrm{d} \psi}{\mathrm{d} s}-\frac{3-n}{3} \psi\right)$ so that in view of $(87 a)$ and $(87 b)$ we have $p \rightarrow 0$ as $s \rightarrow-\infty$.

\section{The solution manifold near the contact line}

In this section, we construct a one-parameter family of solutions to problem $(15 a) \&(15 b)$ through the study of the unstable invariant manifold $\mathcal{M}$ of the dynamical system (95) in the stationary point $(r, \mu, p)=(0,0,0)$.

\section{The dynamic characterization of the unstable manifold}

The linearization of $(95)$ in the stationary point $(0,0,0)$ can be explicitly calculated:

$$
\nabla F(0,0,0)=\left(\begin{array}{ccc}
(3-n) & 0 & 0 \\
0 & 0 & 1 \\
\frac{2(3-n)(2 n-3)}{9} & \frac{(3-n)(2 n-3)}{3} & \frac{4 n-9}{3}
\end{array}\right) .
$$

Its characteristic polynomial reads

$$
\begin{aligned}
P(\zeta) & =(\zeta-(3-n))\left(\zeta^{2}+\frac{9-4 n}{3} \zeta-\frac{(3-n)(2 n-3)}{3}\right) \\
& =(\zeta-(3-n))\left(\zeta+\alpha+3-\frac{4 n}{3}\right)(\zeta-\alpha),
\end{aligned}
$$

where $\alpha$ is given by $(26)$. Since no eigenvalue is zero, the stationary point $(0,0,0)$ is hyperbolic, so that locally, smooth stable and unstable manifolds exist [23]. As two eigenvalues, $\alpha$ and $3-n$, are positive and for $n \in\left(\frac{3}{2}, \frac{7}{3}\right)$ do not coalesce ${ }^{+}$, the tangent space $T_{(0,0,0)} \mathcal{M}$ in $(r, \mu, p)=(0,0,0)$ and the unstable manifold $\mathcal{M}$ are two-dimensional. Furthermore, because the flow $F(r, \mu, p)$ is analytic in a neighborhood of the stationary point $(0,0,0)$, the unstable manifold is locally analytic as well (cf. [11] for a proof). By the dynamic characterization of the unstable manifold $\mathcal{M}$ (globally, it is characterized as the set of all initial data whose solutions backwards in "time" $s$ converge to the stationary point $(0,0,0))$, we must have:

Proposition 7.1. $(r(s), \mu(s), p(s)) \in \mathcal{M}$ for $s \in \mathbb{R}$ for the unique solution of (15).

+ This is the reason, why our considerations are restricted to $n<\frac{7}{3}$, as for $n=\frac{7}{3}$ in fact $\alpha=3-n=\frac{2}{3}$ and the system cannot be diagonalized anymore. 
The geometric characterization of the unstable manifold

We are now ready to prove Proposition 3.2. After the dynamic characterization, we now use the geometric characterization of the unstable manifold: the tangent space $T_{(0,0,0)} \mathcal{M}$ is spanned by the eigenvectors of the positive eigenvalues of $\nabla F(0,0,0), \alpha$ and $3-n$.

Proof of Proposition 3.2. From (96), after straightforward computations we infer that $T_{(0,0,0)} \mathcal{M}$ in $(r, \mu, p)=(0,0,0)$ is determined by

$$
p=\alpha \mu+\frac{2(2 n-3)(3-n-\alpha)}{9(7-3 n)} r \quad \text { with }(\mu, r) \in \mathbb{R}^{2} .
$$

Hence, the unstable manifold $\mathcal{M}$ can be locally in a neighborhood $U \subset \mathbb{R}^{2}$ of $(r, \mu)=(0,0)$ written as a graph $p=P_{n}(\mu, r)$ with $(\mu, r) \in U$ with

$$
P_{n}(0,0)=0, \quad \frac{\partial P_{n}}{\partial \mu}(0,0)=\alpha, \quad \frac{\partial P_{n}}{\partial r}(0,0)=\frac{2(2 n-3)(3-n-\alpha)}{9(7-3 n)} .
$$

The function $P_{n}=P_{n}(\mu, r)$ is analytic in $(\mu, r)$ (cf. [11]) and smoothly depends on the parameter $n^{*}$. Hence, any solution $(r, \mu, p)$ on $\mathcal{M}$ subject to $(r, \mu, p) \rightarrow(0,0,0)$ as $s \rightarrow-\infty$ needs to fulfill $\frac{\mathrm{d} \mu}{\mathrm{d} s}=P_{n}\left(\mu, e^{(3-n) s}\right)$ for $-s \gg 1$. In view of (98) and since by our choice $\alpha<3-n$ (cf. (26)), we expect the asymptotic behavior $\mu=b e^{\alpha s}(1+o(1)$ ) as $s \rightarrow-\infty$ with a parameter $b \in \mathbb{R}$. Setting

$$
y:=e^{\alpha s},
$$

we are lead to consider the ODE

$$
y \frac{\mathrm{d} \mu}{\mathrm{d} y}=\frac{1}{\alpha} P_{n}\left(\mu, y^{\gamma}\right) \quad \text { for } 0<y \ll 1 \quad \text { subject to } \mu \rightarrow 0 \quad \text { as } y \searrow 0,(100)
$$

where $\gamma:=\frac{3-n}{\alpha}$. Note that $3-n>\alpha$, hence $\gamma>1$, for $n<\frac{7}{3}$.

\section{Reformulation as a fixed-point problem}

We may reformulate (100) as a fixed-point problem by introducing the kernel

$$
q(\mu, r, n):=\frac{1}{\alpha} P_{n}(\mu, r)-\mu
$$

so that, by (98),

$$
q(0,0, n)=\frac{\partial q}{\partial \mu}(0,0, n)=0
$$

and (100) takes the form

$$
y \frac{\mathrm{d} \mu}{\mathrm{d} y}-\mu=q\left(\mu, y^{\gamma}, n\right) \quad \text { for } 0<y \ll 1 \quad \text { subject to } \mu \rightarrow 0 \quad \text { as } y \searrow 0 .
$$

* A proof in the case of discrete dynamical systems can be found in [33, App. I], where the smooth dependence of the invariant manifold on parameters is shown, provided that the flow of the system depends smoothly on the latter. 
Integration yields

$$
\mu_{b}(y)=b y+\int_{0}^{1} \sigma^{-2} q\left(\mu(y \sigma), y^{\gamma} \sigma^{\gamma}, n\right) \mathrm{d} \sigma \quad \text { for } 0<y \ll 1,
$$

where $b \in \mathbb{R}$ is a free parameter. By setting

$$
\theta_{b}(y):=\mu_{b}(y)-b y
$$

and defining

$$
S_{\Theta} \phi(y):=\int_{0}^{1} \sigma^{-2} \phi(y \sigma) \mathrm{d} \sigma \quad \text { and } \quad \mathcal{F}[\theta, b, n]:=S_{\Theta} q
$$

with $q=q\left(\theta(y)+b y, y^{\gamma}, n\right)$, we arrive at the fixed-point problem

$$
\theta_{b}=\mathcal{F}\left[\theta_{b}, b, n\right] \text {. }
$$

It remains to endow (104) with a functional-analytic framework that allows to apply Banach's fixed-point theorem (to construct a solution) and the implicit function theorem (to derive the $C^{1}$-dependence on the data). Therefore, we set

$$
\begin{aligned}
& \Theta:=\left\{\theta:\|\theta\|_{\Theta}<\infty\right\} \text { and } N_{\Theta}:=\left\{\theta:\|\theta\|_{\Theta} \leq \Theta_{0}\right\}, \\
& \text { with }\|\theta\|_{\Theta}:=\max _{0 \leq y \leq y_{0}} y^{-1}|\theta(y)|
\end{aligned}
$$

where $y_{0} \in(0,1]$ is such that we can use the graph $P_{n}$. The values of $\Theta_{0}$ and $y_{0}\left(y_{0}\right.$ sufficiently small) will be chosen below, in this order. We also use the norm

$$
\begin{aligned}
\|q\|_{Q}:= & \max _{\substack{0 \leq \mu \leq\left(\Theta_{0}+b_{0}\right) y_{0} \\
0 \leq r \leq y_{0}^{\gamma} \\
n \in I}}^{j=0,1,2} \\
& \left.\left|\partial_{\mu} \partial_{r} \partial_{n}^{j} q(\mu, r, n)\right|,\left|\partial_{r}^{2} \partial_{n}^{j} q(\mu, r, n)\right|\right\},
\end{aligned}
$$

where $I \Subset\left(\frac{3}{2}, \frac{7}{3}\right)$ and $b_{0}>0$. Then it remains to show that for fixed $I \Subset\left(\frac{3}{2}, \frac{7}{3}\right)$ and $b_{0}>0$ there exist $\Theta_{0}>0$ and $y_{0}>0$ with $y_{0} \ll\left(1+b_{0}\right)^{-1}$ and

(a) $\mathcal{F} \in C^{1}\left(N_{\Theta} \times\left[-b_{0}, b_{0}\right] \times I ; \Theta\right)$;

(b) The derivative $\partial_{\Theta} \mathcal{F}$ has uniformly bounded operator norm

$$
\left\|\partial_{\Theta} \mathcal{F}[\theta, b, n]\right\|_{\Theta \rightarrow \Theta} \leq \frac{1}{2} \text { for }(\theta, b, n) \in N_{\Theta} \times\left[-b_{0}, b_{0}\right] \times I,
$$

so that in particular $\operatorname{id}_{\Theta}-\partial_{\Theta} \mathcal{F}[\cdot, b, n]$ is for fixed $(b, n) \in\left[-b_{0}, b_{0}\right] \times I$ an isomorphism in $\Theta$ with uniform bound

$$
\left\|\left(\operatorname{id}_{\Theta}-\partial_{\Theta} \mathcal{F}[\theta, b, n]\right)^{-1}\right\|_{\Theta \rightarrow \Theta} \leq 2 \text { for }(\theta, b, n) \in N_{\Theta} \times\left[-b_{0}, b_{0}\right] \times I ;(108
$$

(c) $\mathcal{F}[\cdot, b, n]$ is a self-map in $N_{\Theta}$ for $(b, n) \in\left[-b_{0}, b_{0}\right] \times I$ fixed.

As in the proof of Proposition 5.1, (a)-(c) imply that for any $I \Subset\left(\frac{3}{2}, \frac{7}{3}\right)$ and any $b_{0}>0$ there exists a $C^{1}$-solution map $\left[-b_{0}, b_{0}\right] \times I \ni(b, n) \mapsto \theta_{b} \in N_{\Theta}$. 
Rigorous asymptotics to the thin-film equation and Tanner's law

Bound on $S_{\Theta}$

We can directly infer from (103)

$$
\left|S_{\Theta} \phi(y)\right| \leq \int_{0}^{1} \sigma^{-2}|\phi(y \sigma)| \mathrm{d} \sigma \leq y^{\delta+1} \delta^{-1} \max _{0 \leq y^{\prime} \leq y_{0}}\left(y^{\prime}\right)^{-1-\delta}\left|\phi\left(y^{\prime}\right)\right|
$$

for $\delta>0$, so that

$$
\left\|S_{\Theta} \phi\right\|_{\Theta} \leq \frac{y_{0}^{\delta}}{\delta} \max _{0 \leq y \leq y_{0}} y^{-1-\delta}|\phi(y)| \quad \text { for all } \delta>0 .
$$

Self-map (proof of (c))

We notice

$$
q(\mu, r, n) \stackrel{(101)}{=} \int_{0}^{\mu} \int_{0}^{\mu^{\prime}} \partial_{\mu}^{2} q\left(\mu^{\prime \prime}, 0, n\right) \mathrm{d} \mu^{\prime \prime} \mathrm{d} \mu^{\prime}+\int_{0}^{r} \partial_{r} q\left(\mu, r^{\prime}, n\right) \mathrm{d} r^{\prime},
$$

so that we may estimate

$$
\begin{aligned}
& \|\mathcal{F}[\theta, b, n]\|_{\Theta} \stackrel{(103),(109)}{\leq}\left(y_{0} \max _{\substack{0 \leq y \leq y_{0} \\
0 \leq \mu \leq\left(\Theta_{0}+b_{0}\right) y_{0}}} \frac{|\theta(y)+b y|^{2}}{y^{2}}\left|\partial_{\mu}^{2} q(\mu, 0, n)\right|\right. \\
& \left.+\frac{y_{0}^{\gamma-1}}{\gamma-1} \max _{\substack{0 \leq y \leq y_{0} \\
0 \leq r \leq y_{0}^{\gamma}}}\left|\partial_{r} q(\theta(y)+b y, r, n)\right|\right) \\
& \stackrel{(105)}{\lesssim} \quad\left(y_{0}\left(\Theta_{0}^{2}+b_{0}^{2}\right)+\frac{y_{0}^{\gamma-1}}{\gamma-1}\right)\|q\|_{Q},
\end{aligned}
$$

where we have used $\delta=1$ and $\delta=\gamma-1>0$ for (109), respectively. Hence, under the assumption that

$$
\left(y_{0}\left(\Theta_{0}^{2}+b_{0}^{2}\right)+\frac{y_{0}^{\gamma-1}}{\gamma-1}\right) \frac{\|q\|_{Q}}{\Theta_{0}} \ll 1 \quad \text { for } n \in I,
$$

indeed $\mathcal{F}[\cdot, b, n]$ maps $\Theta$ into itself.

Lipschitz bound (proof of (b))

We notice that the formal derivative $\partial_{\Theta} \mathcal{F}[\theta, b, n]$ is given by

$$
\partial_{\Theta} \mathcal{F}[\theta, b, n] \partial \theta=\left(S_{\Theta} \partial_{\mu} q\right) \partial \theta,
$$

so that as above we may estimate

$$
\begin{aligned}
& \left\|\partial_{\Theta} \mathcal{F}[\theta, b, n] \partial \theta\right\|_{\Theta} \\
& \stackrel{(109)}{\leq}\left(\max _{0} \max _{\substack{0 \leq y \leq y_{0} \\
0 \leq \mu \leq\left(\Theta_{0}+b_{0}\right) y_{0}}} y^{-2}\left|\partial_{\mu}^{2} q(\mu, 0, n)\right||\theta(y)+b y||\partial \theta(y)|\right.
\end{aligned}
$$


Rigorous asymptotics to the thin-film equation and Tanner's law

$$
\begin{gathered}
\left.+\frac{y_{0}^{\gamma}}{\gamma} \max _{\substack{0 \leq y \leq y_{0} \\
0 \leq r \leq y_{0}^{\gamma}}} y^{-1}\left|\partial_{\mu} \partial_{r} q(\theta(y)+b y, r, n)\right||\partial \theta(y)|\right) \\
\stackrel{(105)}{\lesssim}\left(y_{0}\left(\Theta_{0}+b_{0}\right)+\frac{y_{0}^{\gamma}}{\gamma}\right)\|q\|_{Q}\|\partial \theta\|_{\Theta} \cdot
\end{gathered}
$$

Hence, the Lipschitz bound for $\partial_{\Theta} \mathcal{F}[\theta, b, n]$ is true provided

$$
\left(y_{0}\left(\Theta_{0}+b_{0}\right)+\frac{y_{0}^{\gamma}}{\gamma}\right)\|q\|_{Q} \ll 1 \text {. }
$$

\section{Smallness conditions}

As no further conditions will appear on $\Theta_{0}$ and $y_{0}$, we now discuss (111) and (113). We first choose $\Theta_{0}=1+b_{0}$, so that

$$
\begin{aligned}
& \left(y_{0}\left(\Theta_{0}^{2}+b_{0}^{2}\right)+\frac{y_{0}^{\gamma-1}}{\gamma-1}\right) \frac{\|q\|_{Q}}{\Theta_{0}} \lesssim\left(\left(1+b_{0}\right) y_{0}+\frac{y_{0}^{\gamma-1}}{\gamma-1}\right)\|q\|_{Q}, \\
& \left(y_{0}\left(\Theta_{0}+b_{0}\right)+\frac{y_{0}^{\gamma}}{\gamma}\right)\|q\|_{Q} \lesssim\left(\left(1+b_{0}\right) y_{0}+\frac{y_{0}^{\gamma}}{\gamma}\right)\|q\|_{Q} .
\end{aligned}
$$

We finally choose $y_{0}$ so small that

$$
\left(\left(1+b_{0}\right) y_{0}+\frac{y_{0}^{\gamma-1}}{\gamma-1}+\frac{y_{0}^{\gamma}}{\gamma}\right)\|q\|_{Q} \ll 1,
$$

which is true for $y_{0} \ll\left(1+b_{0}\right)^{-1}$, as the maximum in $\|\cdot\|_{Q}$ is taken on (cf. (106))

$$
(\mu, r, n, j) \in\left[0,\left(\Theta_{0}+b_{0}\right) y_{0}\right] \times\left[0, y_{0}^{\gamma}\right] \times I \times\{1,2,3\},
$$

where $\left(\Theta_{0}+b_{0}\right) y_{0} \lesssim\left(1+b_{0}\right) y_{0} \lesssim 1$ and $y_{0}^{\gamma} \lesssim 1$.

Continuous differentiability (proof of $(a)$ )

We can also identify the other formal derivatives

$$
\partial_{b} \mathcal{F}[\theta, b, n]=S_{\Theta}\left(y \partial_{\mu} q\right) \quad \text { and } \quad \partial_{n} \mathcal{F}[\theta, b, n]=S_{\Theta}\left(\left(\partial_{n} \gamma\right) y^{\gamma} \ln y \partial_{r} q+\partial_{n} q\right) .
$$

For the proof of (a) we merely need to show boundedness and continuity of the directional derivatives with respect to $\theta=\theta(y), b$, and $n$. Boundedness of $\partial_{\Theta} \mathcal{F}[\theta, b, n]$ has been already shown in the previous step. Furthermore, as in (112)

$$
\left\|\partial_{b} \mathcal{F}[\theta, b, n]\right\|_{\Theta} \stackrel{(109)}{\lesssim}\left(y_{0}\left(\Theta_{0}+b_{0}\right)+\frac{y_{0}^{\gamma}}{\gamma}\right)\|q\|_{Q}
$$

and

$$
\left\|\partial_{n} \mathcal{F}[\theta, b, n]\right\|_{\Theta} \stackrel{(109)}{\lesssim}\left(\left|\partial_{n} \gamma\right|\left|\ln y_{0}\right| \frac{y_{0}^{\gamma-1}}{\gamma-1}+y_{0}\left(\Theta_{0}^{2}+b_{0}^{2}\right)+\frac{y_{0}^{\gamma-1}}{\gamma-1}\right)\|q\|_{Q}
$$

where $S_{\Theta} \partial_{n} q$ can be treated as in (110) (the boundary values in (101) do not change under differentiation with respect to $n)$. This demonstrates boundedness of $\partial_{b} \mathcal{F}[\theta, b, n]$ and $\partial_{n} \mathcal{F}[\theta, b, n]$. 
Rigorous asymptotics to the thin-film equation and Tanner's law

For the continuity claim, observe

$$
\begin{aligned}
& \left\|\left(\partial_{\Theta} \mathcal{F}\left[\theta_{1}, b_{1}, n_{1}\right]-\partial_{\Theta} \mathcal{F}\left[\theta_{2}, b_{2}, n_{2}\right]\right) \partial \theta\right\|_{\Theta} \\
& \stackrel{(109)}{=} y_{\Theta}\left(\| S_{\Theta}\left(\partial_{\mu} q\left(\theta_{1}+b_{1} y, y^{\gamma_{1}}, n_{1}\right)-\partial_{\mu} q\left(\theta_{2}+b_{2} y, b_{1}-b_{2} \mid\right)\|q\|_{Q}\|\partial \theta\|_{\Theta}\right.\right. \\
& \quad+\left(\max _{n \in I} \frac{y_{0}^{\gamma}}{\gamma}\left|\ln y_{0}\right|\left|\partial_{n} \gamma\right|\right)\left|n_{1}-n_{2}\right|\|q\|_{Q}\|\partial \theta\|_{\Theta} \\
& \quad+\left(y_{0}\left(\Theta_{0}+b_{0}\right)+\max _{n \in I} \frac{y_{0}^{\gamma}}{\gamma}\right)\left|n_{1}-n_{2}\right|\|q\|_{Q}\|\partial \theta\|_{\Theta},
\end{aligned}
$$

where the last summand, associated to $\partial_{\mu} \partial_{n} q$, is estimated via

$$
\left|n_{1}-n_{2}\right| \max _{n}\left\|\partial_{\mu} \partial_{n} q \partial \theta\right\|_{\Theta} \lesssim\left|n_{1}-n_{2}\right| \max _{n, y}\left|\partial_{\mu} \partial_{n} q\right|\|\partial \theta\|_{\Theta}
$$

and $\max _{n, y}\left|\partial_{\mu} \partial_{n} q\right|$ can be treated as in (110) (the boundary values in (101) do not change under differentiation w.r.to $n$ ). This demonstrates that $\partial_{\Theta} \mathcal{F} \in$ $C^{0}\left(N_{\Theta} \times\left[-b_{0}, b_{0}\right] \times I ; \operatorname{Lin}(\Theta ; \Theta)\right) \sharp$.

By the same reasoning,

$$
\begin{aligned}
& \left\|\left(\partial_{b} \mathcal{F}\left[\theta_{1}, b_{1}, n_{1}\right]-\partial_{b} \mathcal{F}\left[\theta_{2}, b_{2}, n_{2}\right]\right)\right\|_{\Theta} \\
& \left.\stackrel{\stackrel{109)}{=}}{\leq}\left(S_{\Theta} y\left(\partial_{\mu} q\left(\theta_{1}+b_{1} y, y^{\gamma_{1}}, n_{1}\right)-\theta_{2} \|_{\Theta}+y_{0}\left|b_{1}-b_{2}\right|+\left(\max _{n \in I} \frac{y_{0}^{\gamma}}{\gamma}\left|\ln y_{0}\right|\left|\partial_{n} \gamma\right|\right)\left|n_{1}-n_{2}\right|\right)\|q\|_{Q}, n_{2}\right)\right) \|_{\Theta} \\
& \quad+\left(y_{0}\left(\Theta_{0}+b_{0}\right)+\max _{n \in I} \frac{y_{0}^{\gamma}}{\gamma}\right)\left|n_{1}-n_{2}\right|\|q\|_{Q},
\end{aligned}
$$

showing $\partial_{b} \mathcal{F} \in C^{0}\left(N_{\Theta} \times\left[-b_{0}, b_{0}\right] \times I ; \Theta\right)$, and

$$
\begin{aligned}
\|\left(\partial_{n} \mathcal{F}\right. & {\left.\left[\theta_{1}, b_{1}, n_{1}\right]-\partial_{n} \mathcal{F}\left[\theta_{2}, b_{2}, n_{2}\right]\right) \|_{\Theta} } \\
\leq \quad & \| S_{\Theta} \ln y\left(\left(\partial_{n} \gamma_{1}\right) y^{\gamma_{1}} \partial_{r} q\left(\theta_{1}+b_{1} y, y^{\gamma_{1}}, n_{1}\right)\right. \\
& \left.-\left(\partial_{n} \gamma_{2}\right) y^{\gamma_{2}} \partial_{r} q\left(\theta_{2}+b_{2} y, y^{\gamma_{2}}, n_{2}\right)\right) \|_{\Theta} \\
& +\left\|S_{\Theta}\left(\partial_{n} q\left(\theta_{1}+b_{1} y, y^{\gamma_{1}}, n_{1}\right)-\partial_{n} q\left(\theta_{2}+b_{2} y, y^{\gamma_{2}}, n_{2}\right)\right)\right\|_{\Theta} \\
\stackrel{(109)}{\lesssim} \max _{n \in I}\left(\left|\ln y_{0}\right|^{2}\left|\partial_{n} \gamma\right|^{2}+\left|\ln y_{0}\right|\left|\partial_{n}^{2} \gamma\right|+\left|\ln y_{0}\right|\left|\partial_{n} \gamma\right|\right) \frac{y_{0}^{\gamma-1}}{\gamma-1}\|q\|_{Q}\left|n_{1}-n_{2}\right| & \\
& +\max _{n \in I}\left|\ln y_{0}\right|^{2}\left|\partial_{n} \gamma\right|^{2} \frac{y_{0}^{2 \gamma-1}}{2 \gamma-1}\|q\|_{Q}\left|n_{1}-n_{2}\right| \\
& +\max _{n \in I}\left|\ln y_{0}\right|\left|\partial_{n} \gamma\right| \frac{y_{0}^{\gamma}}{\gamma}\|q\|_{Q}\left(\left\|\theta_{1}-\theta_{2}\right\|_{\Theta}+\left|b_{1}-b_{2}\right|\right) \\
& +y_{0}\left(\Theta_{0}+b_{0}\right)\|q\|_{Q}\left(\left\|\theta_{1}-\theta_{2}\right\|_{\Theta}+\left|b_{1}-b_{2}\right|\right) \\
& +\left(\max _{n \in I}\left(\left|\ln y_{0}\right|\left|\partial_{n} \gamma\right|+1\right) \frac{y_{0}^{\gamma-1}}{\gamma-1}+y_{0}\left(\Theta_{0}^{2}+b_{0}^{2}\right)\right)\|q\|_{Q}\left|n_{1}-n_{2}\right|,
\end{aligned}
$$

from which $\partial_{n} \mathcal{F} \in C^{0}\left(N_{\Theta} \times\left[-b_{0}, b_{0}\right] \times I ; \Theta\right)$ follows.

$\sharp \operatorname{Lin}(\Theta ; \Theta)$ denotes the space of linear bounded operators $\Theta \rightarrow \Theta$. 
Proof of (31b) and (31c)

Estimate (110) (with $y_{0}$ replaced by $y$ ) implies that $\theta_{b}(y)=O\left(y^{\kappa}\right)$ as $y \searrow 0$, where $\kappa:=\min \{1, \gamma-1\}$. Therefore $\mu_{b}(y)=b y\left(1+O\left(y^{\kappa}\right)\right)$ as $y \rightarrow 0$ and $(31 b)$ follows from $y \stackrel{(92),(99)}{=} H^{\alpha}$. By differentiating the fixed-point equation (104) with respect to $b$, we obtain

$$
\partial_{b} \theta_{b}=\left(\operatorname{id}_{\Theta}-\partial_{\Theta} \mathcal{F}\left[\theta_{b}, b, n\right]\right)^{-1} \partial_{b} \mathcal{F}\left[\theta_{b}, b, n\right],
$$

so that we can infer

$$
\left\|\partial_{b} \theta_{b}\right\|_{\Theta} \stackrel{(108)}{\leq} 2\left\|\partial_{b} \mathcal{F}\left[\theta_{b}, b, n\right]\right\|_{\Theta} \stackrel{(114)}{\lesssim}\left(y_{0}\left(\Theta_{0}^{2}+b_{0}^{2}\right)+\frac{y_{0}^{\gamma}}{\gamma}\right)\|q\|_{Q} .
$$

This implies $\partial_{b} \theta_{b}=O\left(y^{2}\right)$ as $y \searrow 0$, so that by (102) $\partial_{b} \mu_{b}=y(1+O(y))$ as $y \searrow 0$, which due to $y \stackrel{(99)}{=} e^{\alpha s} \stackrel{(92)}{=} H^{\alpha}$ implies $(31 c)$.

\section{Acknowledgments}

We are grateful for discussions with Christian Kühn, Andreas Münch, and Juan J L Velázquez. We also thank Lukas Döring for carefully reading an earlier version of the manuscript. LG and MVG appreciate the kind hospitality of the Max Planck Institute for Mathematics in the Sciences (MPI MIS) in Leipzig. MVG is much obliged to the International Max Planck Research School (IMPRS) of the MPI MIS and the Fields Institute in Toronto for funding. This material is based upon work partially supported by the National Science Foundation under Grant No. NSF DMS-1054115.

\section{References}

[1] L. Ansini and L. Giacomelli. Shear-thinning liquid films: macroscopic and asymptotic behaviour by quasi-self-similar solutions. Nonlinearity, 15(6):2147-2164, 2002.

[2] G. I. Barenblatt, E. Beretta, and M. Bertsch. The problem of the spreading of a liquid film along a solid surface: a new mathematical formulation. Proc. Nat. Acad. Sci. U.S.A., 94(19):1002410030, 1997.

[3] F. Bernis, L. A. Peletier, and S. M. Williams. Source type solutions of a fourth order nonlinear degenerate parabolic equation. Nonlinear Anal., 18(3):217-234, 1992.

[4] M. Bertsch, R. Dal Passo, S. H. Davis, and L. Giacomelli. Effective and microscopic contact angles in thin film dynamics. European Journal of Applied Mathematics, null:181-201, 42000.

[5] S. Boatto, L. P. Kadanoff, and P. Olla. Traveling-wave solutions to thin-film equations. Phys. Rev. E, 48:4423-4431, Dec 1993.

[6] D. Bonn, J. Eggers, J. Indekeu, J. Meunier, and E. Rolley. Wetting and spreading. Rev. Mod. Phys., 81:739-805, May 2009.

[7] R. Buckingham, M. Shearer, and A. Bertozzi. Thin film traveling waves and the Navier slip condition. SIAM J. Appl. Math., 63(2):722-744, 2002.

[8] M. Chiricotto and L. Giacomelli. Droplets spreading with contact-line friction: lubrication approximation and traveling wave solutions. Commun. Appl. Ind. Math., 2(2):e-388, 16, 2011.

[9] M. Chiricotto and L. Giacomelli. Scaling laws for droplets spreading under contact-line friction. Commun. Math. Sci., 11(2):361-383, 2013. 
[10] H. Chun and L. E. Scriven. Hydrodynamic model of steady movement of a solid/liquid/fluid contact line. Journal of Colloid and Interface Science, 35(1):85 - 101, 1971.

[11] E. A. Coddington and N. Levinson. Theory of ordinary differential equations. McGraw-Hill Book Company, Inc., New York-Toronto-London, 1955.

[12] R. G. Cox. The dynamics of the spreading of liquids on a solid surface. part 1. viscous flow. Journal of Fluid Mechanics, 168:169-194, 71986.

[13] P. G. de Gennes. Wetting: statics and dynamics. Rev. Mod. Phys., 57:827-863, Jul 1985.

[14] B. R. Duffy and S. K. Wilson. A third-order differential equation arising in thin-film flows and relevant to Tanner's law. Appl. Math. Lett., 10(3):63-68, 1997.

[15] E. B. Dussan V. The moving contact line: the slip boundary condition. Journal of Fluid Mechanics, 77:665-684, 101976.

[16] E. B. Dussan V and S. H. Davis. On the motion of a fluid-fluid interface along a solid surface. Journal of Fluid Mechanics, 65:71-95, 81974.

[17] J. Eggers and H. A. Stone. Characteristic lengths at moving contact lines for a perfectly wetting fluid: the influence of speed on the dynamic contact angle. Journal of Fluid Mechanics, 505:309$321,32004$.

[18] P. Ehrhard and S. H. Davis. Non-isothermal spreading of liquid drops on horizontal plates. Journal of Fluid Mechanics, 229:365-388, 81991.

[19] L. Giacomelli, M. V. Gnann, and F. Otto. Regularity of source-type solutions to the thin-film equation with zero contact angle and mobility exponent between $3 / 2$ and 3. European J. Appl. Math., 24(5):735-760, 2013.

[20] L. Giacomelli and F. Otto. Droplet spreading: intermediate scaling law by PDE methods. Comm. Pure Appl. Math., 55(2):217-254, 2002.

[21] L. Giacomelli and F. Otto. Rigorous lubrication approximation. Interfaces Free Bound., 5(4):483$529,2003$.

[22] P. J. Haley and M. J. Miksis. The effect of the contact line on droplet spreading. Journal of Fluid Mechanics, 223:57-81, 21991.

[23] P. Hartman. Ordinary differential equations, volume 38 of Classics in Applied Mathematics. Society for Industrial and Applied Mathematics (SIAM), Philadelphia, PA, 2002. Corrected reprint of the second (1982) edition [Birkhäuser, Boston, MA; MR0658490 (83e:34002)], With a foreword by Peter Bates.

[24] L. M. Hocking. The spreading of a thin drop by gravity and capillarity. The Quarterly Journal of Mechanics and Applied Mathematics, 36(1):55-69, 1983.

[25] L. M. Hocking. Rival contact-angle models and the spreading of drops. Journal of Fluid Mechanics, 239:671-681, 61992.

[26] W. Jäger and A. Mikelić. On the roughness-induced effective boundary conditions for an incompressible viscous flow. J. Differential Equations, 170(1):96-122, 2001.

[27] J. King. The spreading of power-law fluids. In A. King and Y. Shikhmurzaev, editors, IUTAM Symposium on Free Surface Flows, volume 62 of Fluid Mechanics and Its Applications, pages 153-160. Springer Netherlands, 2001.

[28] J. King. Thin-film flows and high-order degenerate parabolic equations. In A. King and Y. Shikhmurzaev, editors, IUTAM Symposium on Free Surface Flows, volume 62 of Fluid Mechanics and Its Applications, pages 7-18. Springer Netherlands, 2001.

[29] H. Knüpfer and N. Masmoudi. Darcy flow on a plate with prescribed contact angle: Well-posedness and lubrication approximation. Arch. Rat. Mech. Anal., page to appear.

[30] H. Knüpfer and N. Masmoudi. Well-posedness and uniform bounds for a nonlocal third order evolution operator on an infinite wedge. Comm. Math. Phys., 320(2):395-424, 2013.

[31] C. L. M. H. Navier. Mémoire sur les lois du mouvement des fluides. Mem. Acad. R. Sci. Paris, 6:389-416, 1823.

[32] A. Oron, S. H. Davis, and S. G. Bankoff. Long-scale evolution of thin liquid films. Rev. Mod. Phys., 69:931-980, Jul 1997. 
[33] J. Palis and F. Takens. Hyperbolicity and sensitive chaotic dynamics at homoclinic bifurcations, volume 35 of Cambridge Studies in Advanced Mathematics. Cambridge University Press, Cambridge, 1993. Fractal dimensions and infinitely many attractors.

[34] T. Qian, X.-P. Wang, and P. Sheng. A variational approach to moving contact line hydrodynamics. J. Fluid Mech., 564:333-360, 2006.

[35] W. Ren and W. E. Boundary conditions for the moving contact line problem. Physics of Fluids (1994-present), 19(2):-, 2007.

[36] W. Ren and W. E. Derivation of continuum models for the moving contact line problem based on thermodynamic principles. Commun. Math. Sci., 9(2):597-606, 2011.

[37] W. Ren, D. Hu, and W. E. Continuum models for the contact line problem. Physics of Fluids, 22(10):102103+, 2010.

[38] S. D. Taliaferro. A nonlinear singular boundary value problem. Nonlinear Anal., 3(6):897-904, 1979.

[39] L. H. Tanner. The spreading of silicone oil drops on horizontal surfaces. J. Phys. D: Appl. Phys., 12:1473-1484, 1979.

[40] P. A. Thompson and S. M. Troian. A general boundary condition for liquid flow at solid surfaces. Nature, 389(6649):360-362, 091997.

[41] O. V. Voinov. Inclination angles of the boundary in moving liquid layers. Journal of Applied Mechanics and Technical Physics, 18(2):216-222, 1977. 